\title{
EL SER HUMANO Y LA LEÑA EN LA CORDILLERA DE MENDOZA (ARGENTINA) A LO LARGO DEL HOLOCENO: SITIO ARQUEOLÓGICO ARROYO MALO 3
}

\author{
DIEGO ANDREONI" F. Y AYLEN CAPPARELLI"
}

\begin{abstract}
RESUMEN
El objetivo del presente trabajo es caracterizar cuali-cuantitativamente el registro antracológico del sitio arqueológico Arroyo Malo 3, que se emplaza en el valle del río Atuel, Mendoza, y cuya secuencia temporal comprende desde el Holoceno Temprano hasta el Holoceno Tardío. Se pretende establecer si el proceso de intensificación propuesto para los últimos 2200 años de ocupación humana de la región se ve reflejado, de alguna manera u otra, en el registro de uso combustible de las especies leñosas de la zona. De los 6650 carbones de madera recuperados de la cuadrícula B-1 del sitio, se seleccionó una submuestra por cada uno de los 40 niveles excavados. En total se analizaron 701 carbones. Éstos fueron identificados mediante comparación con la colección de referencia elaborada previamente para la zona por los autores. Se identificaron representantes de los siguientes géneros: Adesmia, Anarthrophyllum, Berberis, Colliguaja, Chusquea, Chuquiraga, Ochetophila, Ephedra, Junellia, Lycium, Mulinun, Schinus, Senna y Prosopis. Los resultados muestran cambios en cuanto a presencia/ausencia y frecuencia relativa de los distintos géneros a los largo del tiempo, que tienden hacia una mayor diversificación taxonómica, e incluso incorporación de maderas alóctonas (i.e. Chusquea), hacia el Holoceno Tardío.
\end{abstract}

PALABRAS CLAVE: antracología, intensificación, Patagonia de Mendoza, leña.

\section{HUMANS AND FIREWOOD IN THE ANDEAN MENDOZA (ARGENTINA) ALONG THE HOLOCENE: THE ARCHAEOLOGICAL SITE ARROYO MALO 3}

\begin{abstract}
The objective of the present paper is to characterize quali-quantitatively the anthracological record of the archaeological site Arroyo Malo 3, located in the valley of the Atuel river, Mendoza, whose temporal sequence comprises from the Early to the Late Holocene. We intend to establish if the intensification process proposed for the last 2200 years of the human occupation of the region is reflected, from one or another manner, in the firewood record of the woody plants of the area. From the 6650 wood charcoal remains recovered from the B-1 grid of the site, one subsample of each of the 40 archaeological levels excavated


was selected. A total of 701 remains was analysed. Charcoal was identified by comparison with the reference collection previously made by the authors for the area. The following taxa were identified: Adesmia, Anarthrophyllum, Berberis, Colliguaja Chusquea, Chuquiraga, Ochetophila, Ephedra, Junellia, Lycium, Mulinun, Schinus, Senna and Prosopis. Results showed changes in presence/absence and relative frequence values of the different genera along the temporal sequence of the site, which tend towards a taxonomical diversification, and also to the incorporation of foreign wood (i.e. Chusquea), during the late Holocene.

KEY WORDS: anthracology, intensification, Patagonia of Mendoza, firewood.

\section{INTRODUCCIÓN}

A lo largo de la historia de la especie humana, el hombre ha sabido dar a la vegetación distintos usos, siendo empleada para alimento, medicina, vestimenta, abrigo, combustible, materia prima para confeccionar herramientas, entre muchos otros. Los estudios de macrorrestos vegetales provenientes de sitios arqueológicos han permitido dilucidar dichos usos en el pasado y discutir las características de numerosos procesos relacionados con los mismos, tales como la recolección de especies silvestres, la agricultura, la domesticación, el almacenamiento, entre otros. En el caso de los recursos leñosos, material que nos ocupa en el presente trabajo, los carbones arqueológicos han sido estudiados para evaluar estrategias en el uso de diferentes combustibles, sobreexplotación de recursos, selección de materiales construccionales y maderas empleadas en la confección de instrumentos tanto en el Viejo (i.e. Asouti y Hather 2001; Marston 2009, y Willcox 2000, entre otros) como en el Nuevo Mundo (Capparelli 2004, Capparelli et al. 2009; Caruso et al. 2008; Marconetto 2005; Piqué i Huerta 1999, Solari 1993, entre otros). Particularmente, nos interesa analizar el registro arqueológico de restos de leño carbonizado del sitio Arroyo Malo 3 (AMA-3), en el marco del proceso de intensificación propuesto por Neme (2007, 2009) para los últimos 2200 años de ocupación humana del Sur de Mendoza.

Durante las últimas décadas se ha planteado para algunas áreas arqueológicas de Argentina, entre ellas el sur mendocino, el desarrollo de procesos de intensificación en el aprovechamiento de recursos a lo largo del Holoceno. Los mismos han sido planteados como una de las posibles respuestas a un incremento continuo del número poblacional, y por ende, a una disminución del retorno percápita de recursos, dentro del seno de sociedades cazadoras-recolectoras especializadas en la utilización de un recurso base en una zona ambiental más o menos homogénea. Se llevarían a cabo a través de la inversión de mayor cantidad de energía por cada individuo por unidad de tiempo, lo que redundaría en una mayor productividad por unidad de área (Broughton 1994) y en una disminución del riesgo y la vulnerabilidad de estas sociedades, aumentando el mencionado retorno per capita (Aldendelfer 1998). Estos procesos de intensificación pueden verse reflejados de varias maneras, por ejemplo mediante una diversificación en el uso de recursos, en la aparición de nueva tecnología vinculada a un aprovechamiento más integral de los mismos, en la ocupación y explotación de áreas más pobres, en la regionalización de estilos, en la circulación de bienes a gran escala, en el aumento de la dependencia de recursos almacenables, y/o en la disminución de la movilidad de las sociedades. En el sur de Mendoza se ha propuesto que estos procesos se reflejan en distintos contextos y materiales del registro arqueológico del área a partir de los 2.200 años AP (Neme 2007), incluso se interpreta al poblamiento de La Payuniauna de las principales áreas biogeográficas del sur de Mendoza (Gil 2006)-, como una manifestación de los mismos. Sin embargo, entre los diferentes tipos de registro arqueológico, el del material leñoso aún no había sido estudiado. Es por eso que el presente trabajo tiene por objeto establecer si dicho proceso de intensificación de ocupación humana del sur de Mendoza se ve reflejado, de alguna manera u otra, en el registro de uso combustible de las especies leñosas de la zona. Este objetivo se lleva a cabo a través de la caracterización cuali-cuantitativa del conjunto antracológico del sitio Arroyo Malo 3 (AMA-3) 3 .

3 A nivel macrorregional, el presente trabajo se enmarca en un proyecto mayor dirigido por la Dra. Capparelli, en el cual nos interesa conocer de qué modo se pudieron haber dado los procesos de intensificación de recursos vegetales a través del tiempo a lo largo de la denominada Diagonal Árida Argentina. 


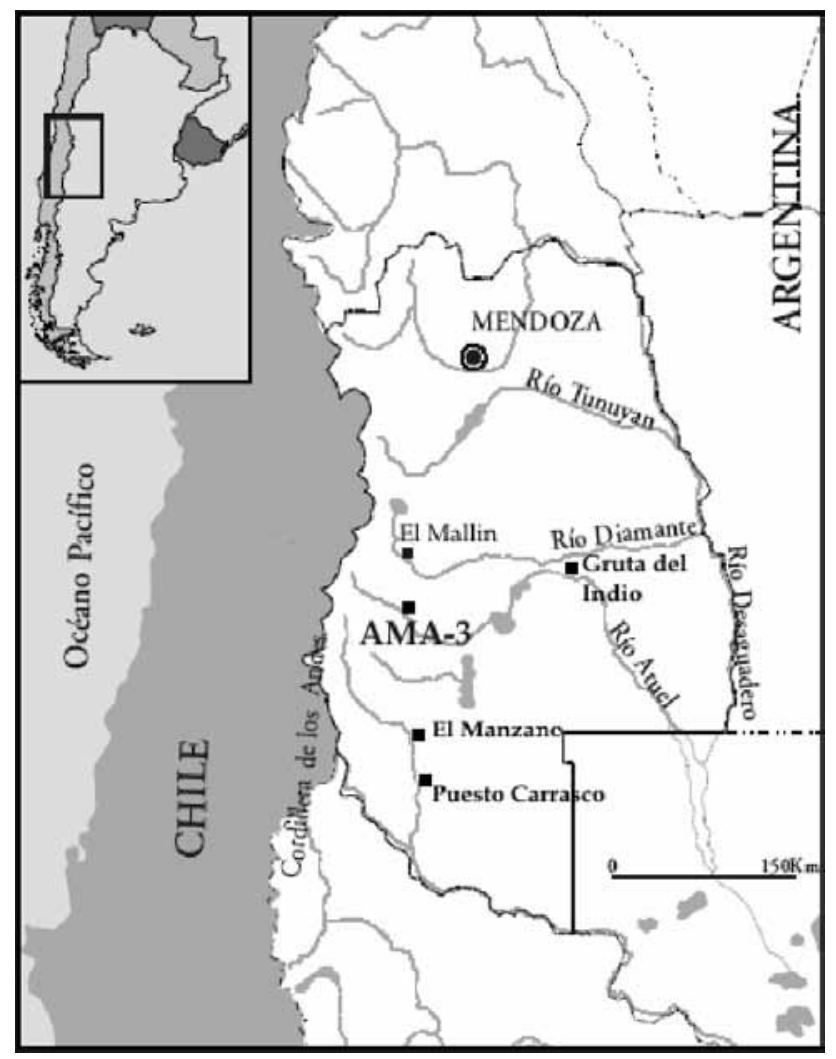

Fig 1. Ubicación del sitio AMA-3 y de otros sitios mencionados en el trabajo

Se entiende que el proceso de intensificación mencionado podría ser potencialmente evidenciado en el registro leñoso a través de una diversificación de especies leñosas utilizadas como combustible a lo largo del tiempo, por la incorporación de especies foráneas, por la sobreexplotación de taxa de buena calidad combustible (puede implicar sobreexplotación numérica o la aparición de estructuras anatómicas que impliquen el aprovechamiento no solo de la parte aérea de la planta sino también de su raíz, con la consecuente muerte de la planta en cuestión), o por la gestión de recursos leñosos de baja calidad.

\section{ÁREA DE ESTUDIO Y ANTECEDENTES ARQUEOLÓGICOS DEL SITIO AMA-3}

El sitio AMA-3 es un alero de andesita perteneciente a la formación cerro Guanaquero (Volkheimer 1978), se ubica en la parte media del valle del río Atuel, en la margen izquierda del arroyo Malo (34 51' 20"' S, 69 53' 15" W), tributario del primero (Fig 1). El alero se ubica a $2000 \mathrm{~m} \mathrm{snm}$ aproximadamente. Este sitio forma parte de un complejo arqueológico mayor dentro del cual se encuentran cinco sitios más (Neme 2007). El alero tiene $13 \mathrm{~m}$ de abertura, 7,5 $\mathrm{m}$ de profundidad y 3 $\mathrm{m}$ de altura, mira hacia el sur en un valle que posee una orientación este-oeste. La superficie excavada del mismo fue de $6 \mathrm{~m}^{2}$ (Dieguez y Neme 2003). El sitio AMA-3 ha sido objeto de trabajos sistémicos los cuales se realizaron en dos etapas. En la primera etapa, entre enero de 1995 y febrero de 1996, se excavó la cuadrícula A-1 (Fig 2), de $2 \times 1 \mathrm{~m}$, en el centro del alero, $y$ al oeste de un sondeo realizado en 1992. En la misma, se excavaron 35 niveles arbitrarios de $5 \mathrm{~cm}$ hasta alcanzar la roca de base, dejando así expuesta una secuencia de 1,75 m de profundidad. Posteriormente, en el año 2000, se realizó una segunda cuadrícula, llamada $\mathrm{B}-1$, que abarcó $4 \mathrm{~m}^{2}$, de la que se excavaron 40 niveles de $5 \mathrm{~cm}$ cada uno, alcanzándose una profundidad de 2 m. Cada uno de estos niveles fue dividido en cuatro sectores según su ubicación espacial: NE, NW, SE y SW (Dieguez y Neme 2003; Neme 2007). 


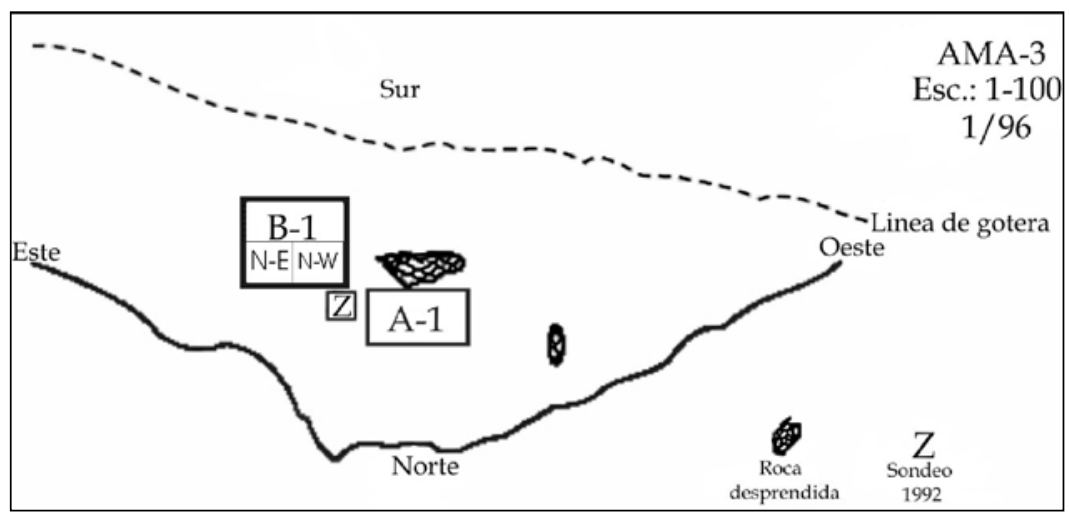

Fig 2. Planta del sitio AMA-3 (modificada de Neme 2007)

La ubicación del sitio debió presentar un atractivo particular para las poblaciones de la región. Por un lado, por la proximidad a una fuente de agua permanente como el arroyo Malo, por otro, por brindar una amplia visión del valle medio del río Atuel, así como por encontrarse al reparo de los vientos predominantes del sector oeste. La ubicación de AMA-3 permite acceder a dos pisos ecológicos y fitogeográficos bien diferenciados como son el del Monte hacia el este del sitio y el Altoandino hacia el oeste, lo cual habría facilitado a la población de cazadores recolectores un rápido acceso a distintos tipos de recursos.

Las condiciones ambientales del Sur de Mendoza han variado desde fines del Pleistoceno hasta la actualidad (Zárate 2002, Zárate et al. 2010). Siguiendo a Zárate (2002), podemos ordenar los cambios paleoambientales en cuatro escenarios que se fueron desarrollando de modo progresivo, configurando estructuras ambientales y fitogeográficas particulares. El Tardiglacial comprende entre los 14.000-10.000 años AP y está caracterizado por una temperatura levemente más elevada que la acontecida durante el Ultimo Máximo Glaciar, pero más fría que la actual. Durante este periodo los glaciares cordilleranos estarían en retroceso. Aunque el registro polínico para este periodo es muy escaso (Páez et al. 2010) en el sur de Mendoza, los análisis realizados en Gruta del Indio por D’Antoni (1983) permiten inferir que, en la planicie oriental, habrían existido precipitaciones invernales, y moderadas temperatura media y aridez el resto del año, con una vegetación dominante de tipo Patagónica. Para el Holoceno Temprano D'Antoni (1983) infiere que, entre los 11.000 años AP y algún momento entre los 9.000/8.000 AP, se habría dado una situación ecotonal entre Monte/Patagonia lo que sugiere un lento incremento en la temperatura y el fin de ciclos de congelamiento y derretimiento del periodo previo (Zárate 2002). El Holoceno Medio, que comprende el periodo entre los 8.000 y 4.000 años AP, se caracteriza por un marcado ascenso de la temperatura y por un proceso de desertificación regional inferido a partir del registro polínico de la Gruta del Indio, aunque existe un hiatus estratigráfico en la secuencia de dicha gruta que no permite establecer claramente la extensión cronológica del mismo (Zárate 2002). Dicha desertificación estaría dada también por una disminución en las lluvias de primavera verano entre el 8.500 y 5.000 AP (ver discusión en Morales et al. 2009). Durante este lapso temporal los registros polínicos permitieron inferir algunas tendencias en la composición vegetal de la región (Páez et al. 2010), que estarían señalando la conformación, entre los 6.000 AP y los 4.000 AP de extensos jarillales de Larrea spp. (Zigophyllaceae) y bosques de Prosopis que se desarrollarían en los valles, con menor proporción de taxa Andino-Patagónico-Puneños. En los ambientes de montaña la aridez se podría ver reflejada en la menor productividad de las vegas. El Holoceno Tardío, que ocurriría aproximadamente a partir de de los 3.000-4.000 años AP hasta la actualidad, coincide con el inicio de condiciones climáticas similares a las actuales (Páez et al. 2010), caracterizadas por una gran variabilidad ambiental y la influencia de los eventos del Niño (Zárate 2002).

Los registros polínicos puntuales más cercanos al sitio AMA-3, el de Agua Buena y el de Laguna El Sosneado (Páez et al. 2010 y Navarro 2010, respectivamente), brindan mayor especificidad geográfica con respecto a parte de los cambios climáticos del área. 
El de Agua Buena permite reconstruir la vegetación a partir de los 4.000 años AP hasta el presente (Páez et al. 2010). Entre los 4.000 y los 2.800 años AP se observó un predominio de taxa Andino-Patagónicos (Mulinum, Azorella, Nassauvia, Colliguaja, Adesmia, Acaena, Fabiana) que disminuirían en cantidad entre los 2.800 y 2.300 años AP, para dar mayor lugar a Asteraceas, Poaceas y halófitas, pero sin desaparecer del todo los primeros. Hacia estos momentos más recientes habría existido también en la cordillera un incremento en el registro polínico de Ephedra que, en términos de abundancia, no posee análogos modernos (Páez et al. 2010). El perfil de El Sosneado se encuentra en los alrededores del lago homónimo y representa características locales diferentes a las registradas en los perfiles antes mencionados. Allí se determinó entre los 6.400 y los 2.150 años AP la existencia de condiciones climáticas moderadas pero más húmedas que las actuales, que permitieron el desarrollo de una estepa arbustiva dominada por Poaceae, Chenopodiaceae, Asteraceae, Mulinum, Ephedra y Schinus, Los altos valores registrados en los taxa acuáticos después de los 6.400 años AP representarían el comienzo de la formación del lago. Entre los 3.200 y los 2.150 años AP los incrementos en los valores de Poaceae, Apiaceae y Mulinum indican un período aún más húmedo que el anterior. Durante este período los taxa acuáticos presentan sus menores valores, por lo cual el lago habría alcanzado sus más altos niveles de agua. Estos registros son coincidentes con los resultados obtenidos a partir de los análisis de micromamíferos de dos sitios arqueológicos del área (AMA-3 y El Sosneado 3) (Neme et al. 2011). Entre los 2.150 y los 600 años AP se registra una vegetación rala y condiciones más áridas que las anteriores, con un incremento en los valores de Chenopodiaceae, Asteraceae, Ephedra y Schinus, entre otros taxa Andino-Patagónicos. Los últimos 600 años AP marcan el desarrollo de condiciones semiáridas semejantes a las actuales y bajos niveles de agua en el lago El Sosneado (Navarro 2010).

Actualmente el clima de la región presenta una temperatura media anual de $10^{\circ} \mathrm{C}$ a $15^{\circ} \mathrm{C}$ en la planicie y de $1^{\circ} \mathrm{C}$ a $5^{\circ} \mathrm{C}$ en la zona de montaña. El régimen de precipitaciones varía también con el gradiente altitudinal: por encima de los $2000 \mathrm{~m}$ snm supera los $1000 \mathrm{~mm}$ anuales, registrándose el $90 \%$ en invierno en forma de nieve; mientras que por debajo de los $2000 \mathrm{~m}$ snm alcanza apenas los $200 \mathrm{~mm}$ anuales (Capitanelli 2005).

Fitogeograficamente, y a una macro-escala, AMA-3 se encuentra en un área ecotonal producida tanto por las variaciones altitudinales como por la orientación y extensión este-oeste del valle. En dicha área se interdigitan las Provincias de Monte, Patagónica y Altoandina (Fig. 3) (Böcher et al. 1972; Cabrera 1976). A una escala regional el sitio se encuentra emplazado en la provincia Patagónica, caracterizada por estepas arbustivas que cuentan con la presencia de especies tales como Colliguaja intergerrima, Anarthrophyllum patagonicum, Chuquiraga oppositifolia, Adesmia pinifolia, entre otras (Böcher et al. 1972). Adesmia pinifolia es remplazada por encima de $2200 \mathrm{msnm}$ por Adesmia obovata (Roig 1972; Roig et al. 2000). Si se disminuye en el gradiente altitudinal hacia el este del valle, la vegetación toma el aspecto de una estepa arbustiva subtropical de monte, donde son frecuentes especies tales como Atriplex lampa, Chuquiraga erinacea, Larrea divaricada, Prosopis alpataco, Bougainvillea spinosa, Lycium chilense y Baccharis angulata, entre otras (Böcher et al. 1972). Por otro lado, a medida que aumenta el gradiente altitudinal y en dirección oeste, aparecen más representadas gramíneas tales como Festuca sp. y Stipa sp., entre otras, y Mulinum sp., en grandes extensiones. La vegetación de tipo Altoandina comienza aproximadamente a los 2400 msnm, y se caracteriza en la región por pastizales, tanto de Poa holciformis, que cubren la mayor parte del sector andino, como de Stipa chrisophylla o S. vaginata, así como también por especies de pulvinadas, entre las que se encuentran Junellia uniflora, Oxalis compacta, y Azorella lycopodioides (Roig et al. 2000).

Desde un punto de vista microregional la vegetación circundante al sitio AMA 3, fue relevada por Hernández (1996) a través de la realización de un site catchment circular de $5 \mathrm{~km}$ de diámetro aproximadamente, análisis que nos permite agregar a los taxa antes mencionados la presencia de Ephedra sp, Schinus polygamus, Senecio multicaules, $S$. subumbellatus, S. gillesii, Taraxacum gilliesi, Hymemoxis haenckeana, Haplopopus sp, Maihuenia sp, Senna arnottiana, Lathyrus sp., Trifolium sp., Cortaderia sp., Stipa sp., Ranunculus sp., Caltha sp., Fabiana sp., Schizantus grahanii, Verbena sp., y Chenopodium sp. Dicha autora considera que los 


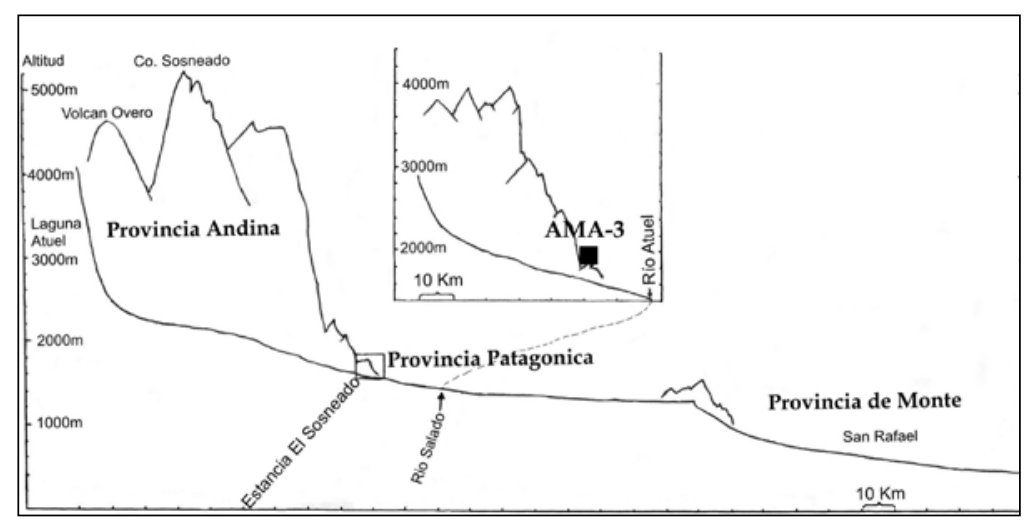

Fig 3. Perfil del valle del Río Atuel (modificado de Bosche 1972)

principales recursos leñosos de la región estarían dados por Schinus sp y Anathrophyllum sp. La información obtenida de dicho site catchment es ampliada por Neme (2007) a partir de los sitios arqueológicos AMA-1 y AMA-3, quien menciona que entre los recursos leñosos la Adesmia es la más abundante, Anarthrophyllum, Senna, Mulinum, Chuquiraga y Schinus son medianamente abundantes, y Senecio, Junellia, Verbena, Fabiana, y Ephedra se encuentran menos representados. Por último, las investigaciones ecológicas realizadas por Böcher et al. (1972), permiten agregar a la lista anterior de especies presentes en la microregión de AMA 3, tanto taxa de filiación Patagónica (i.e. Astephanus fallax, Acantolippia seriphioides, Junellia ligustrina, Azorella guilliesii, Cerastium arvense, Grindelia chilensis, Nassauvia axillaris), como otros que son asignados por los autores a la provincia Altoandina (i.e. Adesmia schneideri), y finalmente otros que caracterizan a provincia Puneña (i.e. Fabiana denudata).

El sitio AMA-3 cuenta con una serie de fechados radiocarbónicos que evidencian su ocupación desde el Holoceno Temprano (8900 AP) hasta el Holoceno Tardío (Tabla 1). En general, los fechados radiocarbónicos muestran una coherencia interna en las dos cuadrículas excavadas, aunque existe un fechado invertido en la cuadricula A-1 (nivel 40 de
4.500 años AP), discordancia que fue interpretada como producto de procesos de formación del registro arqueológico, principalmente debida a la actividad de animales fosoriales, pero también como consecuencia de la formación de los depósitos y de la cubeta sedimentaria (Dieguez y Neme 2003). Este es uno de los motivos por el cual los estudios antracológicos solo se llevaron a cabo con materiales provenientes de la cuadricula B-1 (ver metodología). A partir de las diferentes características del sedimento, Dieguez y Neme (2003) han dividido la secuencia del sitio en tres unidades estratigráficas ( $\mathrm{A}, \mathrm{B}$ y $\mathrm{C}), \mathrm{y}$, sobre la base de los fechados radiocarbónicos, en 4 conjuntos temporales (Tabla 1).

El registro arqueológico de AMA-3 ha sido estudiado prácticamente en su totalidad. El conjunto arqueofaunístico analizado por Neme (2002, 2007), permitió al autor observar cambios en la explotación de la fauna local a lo largo del tiempo. Se evidencia una disminución en la cantidad relativa de camélidos capturados y un aumento en las proporciones de animales de menor tamaño durante los últimos 2200 años AP (Neme 2002). Otro elemento llamativo es la alta tasa de fractura que presentan los huesos, de camélidos principalmente, en los niveles más tardíos, lo que estaría indicando un aprovechamiento intensivo de éstos por medio de la extracción de su médula (Neme y Gil 2002; Neme 2007). El material

Tabla 1. Cronología del sitio AMA-3 (modificada de Neme 2007).

\begin{tabular}{|c|c|c|c|}
\hline Conjuntos & Cronología estipulada & Niveles & Unidades estratigráficas \\
\hline \multirow{2}{*}{1} & $100-2.200 \mathrm{AP}$ & 1 al 9 & $\mathrm{~A}$ \\
\hline 2 & $2.200-5.000 \mathrm{AP}$ & 10 al 12 & $\mathrm{~B}$ \\
\hline 3 & $5.000-7.600 \mathrm{AP}$ & 13 al 22 & $\mathrm{C}$ \\
\hline 4 & $7.600-8.900 \mathrm{AP}$ & 23 al 27 & $\mathrm{C}$ \\
\hline
\end{tabular}


lítico fue estudiado en dos ocasiones, por un lado Neme (2007) analizó los materiales procedentes de la cuadricula A-1 y, más tarde, Pérez (2008) los materiales de la cuadricula contigua B-1. Ambos estudios coinciden en definir una clara tendencia hacia la sustitución de materias primas locales en favor de la incorporación de otras foráneas como sílice y obsidiana, lo que implicaría un aumento en la movilidad de los grupos o en la ampliación de redes de intercambio, ya que las canteras de obsidiana más próximas se encuentra a unos $57 \mathrm{~km}$; tendencia que se acentúa en los componentes más tardíos del sitio (Neme 2007; Pérez 2008), al tiempo que se desarrollan tecnologías más expeditivas. Otro elemento destacable lo constituye la proliferación de instrumental de molienda a partir de los últimos 2.200 años AP (Neme 2007). El análisis de material arqueobotánico correspondiente a carporrestos lo realiza Llano (2005, 2008, 2011), quien identifica semillas desecadas de Chenopodiaceas (Chenopodium sp., C. ambrosioides, C. multifidum y $C$. papulosum) y Cactaceas (Maihuenia sp. y Austrocactus aff. patagonicus), así como restos disecados de Cyperaceas y Asteraceas y semillas carbonizadas de Schinus poligamus. De los cuatro conjuntos temporales analizados, los carporestos vegetales se encuentran solo en los conjuntos 1,2 y 3 , siendo su presencia mayor en el conjunto 1 , correspondiente al Holoceno Tardío. La autora interpreta que estos restos, principalmente las Chenopodiaceas, pueden entenderse como parte de la ampliación de la dieta hacia especies silvestres que hasta el momento habrían tenido poca importancia para los pobladores del sur de Mendoza; las cuales, por su contenido en metabolitos tóxicos para el hombre (saponinas y nitritos), habrían requerido del "mejoramiento de los granos" (sensu López et al. 2011) previo a su consumo (Llano 2008). Este conjunto de datos arqueofaunísticos, líticos y arqueobotánicos avalan la hipótesis propuesta por Neme (2002; 2007) en cuanto al acontecimiento de procesos de intensificación en el sur de Mendoza durante los últimos 2.200 años AP. Finalmente, cabe mencionar que es llamativa la ausencia de material cerámico en el sitio.

Otros estudios realizados son los referidos al registro de microvertebrados, llevados a cabo por Neme et al. (2002, 2011), quienes analizan el origen y la relevancia de dicho tipo de registro, datos que utilizan para realizar y discutir algunas inferencias paleoambientales. En principio estos autores caracterizan y evalúan el origen de los restos estudiados, y llegan a la conclusión de que en ningún caso es posible asignar un origen antrópico a los mismos. Teniendo en cuenta los cambios ambientales conocidos para la región y la distribución de los taxa identificados, Neme et al. (2002) infieren que la presencia de Andalgalomys-especie propia del Dominio Chaqueño- en los componentes más antiguos estudiados (8900 años AP), puede ser correlacionada con un aumento de la temperatura y consecuentemente con un retroceso de las masas glaciares (Markgraf 1983 en Neme et al. 2002) durante ese período. Por otro lado, estos autores consideran que la presencia de micromamiferos-en particular Lestodelphys- en los niveles superiores de la secuencia (3.800 AP), estaría indicando el establecimiento de las condiciones actuales, con un tipo de ambiente patagónico, lo que se ve confirmado por el aumento en la frecuencia relativa de iguánidos a partir de los 4.000 años AP, animales característicos de climas desérticos como el que actualmente presenta la región (Neme et al. 2002).

\section{MATERIALES Y METODOLOGÍA}

Los restos antracológicos del sitio AMA-3 fueron obtenidos durante las excavaciones realizadas entre los años 1996 y 2000, y proceden tanto de la recuperación in situ como de la aplicación de la técnica de zaranda en seco, empleando mallas de $3 \mathrm{~mm}$ de apertura, a la totalidad del sedimento extraído por nivel. Todos los restos antracológicos recuperados corresponden a carbones dispersos en el sedimento del sitio. Tal como se mencionó anteriormente, la determinación anatómica y taxonómica se limitó a la cuadricula B-1, ya que es ésta la que presenta mayor cantidad de restos antracológicos y una secuencia completa y coherente de fechados radiocarbónicos, que abarcan desde el Holoceno Temprano hasta el Tardío (Tabla 1). Dentro de la cuadrícula B-1, fue analizado el sector N (Fig. 2), que abarca una superficie de 2 metros por 1 metro. Dicha elección se basa en dos razones principales: 1- este es el sector de donde mayor cantidad de carbones se ha recuperado; 2 - el sector $\mathrm{N}$ se encuentra a mayor reparo de la lluvia y de los vientos por su ubicación respecto al sector $\mathrm{S}$, lo cual permite minimizar los factores tafonómicos que pudieron haber ocurrido 
dentro del sitio. De cada nivel del sector $\mathrm{N}$ se seleccionó para su análisis el cuadrante (NE o NW) con mayor cantidad de restos antracológicos recuperados (Tabla 2). El único nivel que no pudo ser analizado en su totalidad es el nivel 23, ya que los restos allí presentes fueron utilizados, con anterioridad a este estudio, para fechados radiocarbónicos.

La identificación de los restos fue realizada para cada uno de los 40 niveles artificiales de 5 $\mathrm{cm}$ de espesor de la cuadrícula de excavación (ver sección anterior). Dada la abundancia de restos leñosos recuperados durante la excavación del sitio, de cada uno de los niveles se seleccionó, en el laboratorio, una submuestra para su análisis. La cantidad de carbones de cada submuestra (Tabla 2) fue definida por medio de la construcción de una "curva de riqueza especifica" (también denominada curva de esfuerzo-rendimiento (Solari 2000), técnica mayormente utilizada por los investigadores del área por su operatividad. La misma se basa en la identificación de fragmentos hasta que la curva producida por el número de fragmentos identificados $(\mathrm{X})$ y la aparición de especies nuevas (Y) se hace asintótica a X (Marconetto 2005; Pique i Huerta 1999). Dicha cantidad puede variar de un nivel a otro o dentro en un mismo sitio (Pique i Hueta 1999), según la cantidad de restos presentes y la diversidad específica de la muestra. En cuanto a los tamaños de los restos identificados, se optó por seleccionar alternadamente carbones con dos tamaños distintos: a- entre 3 y 5 $\mathrm{mm}, \mathrm{b}->5 \mathrm{~mm}$, con el fin de no sesgar la muestra a favor de uno u otro tamaño (Miller 1985, Thièbault 1989). Todos los carbones fueron fracturados a mano para el reconocimiento de los rasgos diagnósticos en corte transversal (CT) y longitudinales tangencial (CLT) y radial (CLR), que se realizó en primera instancia en Lupa Binocular Nikon SMZ-100, con aumentos de entre 10X a 160X. En una segunda instancia, y para confirmar la identificación, se utilizó un Microscopio Óptico Nikon eclipse 80i, adaptado con luces alógenas externas, que permitió alcanzar aumentos de hasta 200X.

Para llevar a cabo la identificación de los restos antracológicos se elaboró una colección de referencia de las plantas leñosas del área de estudio, de la cual se obtuvieron ejemplares de herbario y muestras de madera. De las muestras de madera se extrajeron dos cubos de $1,5 \mathrm{~cm}$ de lado. El primero fue utilizado para la realización de los cortes histo- lógicos delgados en los tres planos de observación de la madera (CT, CLT y CLR) (para mayor detalle ver Andreoni 2010), a partir del cual se describieron los rasgos diagnósticos de cada taxa colectado -en el presente trabajo se presentan sólo aquellos de los taxa que no fueron descriptos en Andreoni (2010)-. Las descripciones anatómicas se realizaron siguiendo los criterios cualitativos de IAWA (1989) para plantas dicotiledóneas, y de IAWA (2004) para la único género de Gimnosperma, Ephedra sp. El segundo cubo de madera fue utilizado para realizar carbonizaciones de referencia (ver Andreoni et al. 2011 para un mayor detalle en la metodología y características de cada una de las especies después de la carbonización). Es sabido que durante el proceso de carbonización se producen modificaciones de las características morfológicas cuantitativas de cada taxa, no obstante los rasgos cualitativos propios se conservan (Rossen y Olson 1985; Solari 2000), por lo tanto, fueron estos últimos los principales rasgos diagnósticos utilizados para la identificación taxonómica de los restos leñosos arqueológicos del presente trabajo, que sirvieron para la elaboración de una clave dicotómica que se describe en el acápite siguiente.

Los parámetros cuantitativos más comúnmente utilizados para el análisis de las asociaciones arqueobotánicas son los conteos del número absoluto de restos, el peso, el cálculo de las densidades (número de restos por cantidad de sedimento procesado) y la ubicuidad (porcentaje de las muestras en que cada taxon está presente) (Capparelli 2009, Smart y Hoffman 1988). En el presente trabajo se calculó, para cada taxon en particular, su cantidad absolutapor nivel, conjunto y total-, la frecuencia relativa $\%$ (basada en la variable anterior) y la ubicuidad. La utilización de la cantidad absoluta de fragmentos, en lugar de la densidad, se justifica porque la cantidad de sedimento procesada de cada sector en cada nivel fue la misma a lo largo de todo perfil de excavación. El peso se calculó en cada nivel, conjunto temporal y sitio como un todo, tanto para la muestra total de restos antracológicos recuperada del sitio, como para la submuestra de taxa identificados.

Se sabe que la representatividad diferencial del los taxa en el registro arqueológico está condicionada, además de por las prácticas culturales pasadas de uso de leña, por patrones que tienen que ver con respuestas diferenciales: 1- al proceso 
de carbonización en sí mismo (dadas, entre otros factores, por las condiciones de dicho proceso y por distintas características intrínsecas de la madera de uno $\mathrm{u}$ otro taxon), 2- a procesos posdeposicionales (fragmentación, migración), y 3- a factores posrecuperación de los restos (Chabal 1988, 1990, 1992; Théry-Parisot et al 2010, Piqué i Huerta 1999). De aquellos que tienen que ver con el proceso de combustión en sí mismo, se caracterizaron, en trabajos anteriores, el efecto de variables tales como la temperatura, la humedad y la densidad de los taxa aquí estudiados (Andreoni et al. 2010), resultados que se cruzan con los del presente trabajo en la discusión. Con respecto al sesgo producido por los patrones de fragmentación, se sabe que diferentes taxa, luego de su carbonización en condiciones semejantes, presentan patrones de fragmentación similares (Chabal 1988, 1990, 1992 y Théry-Parisot et al. 2010, entre otros), lo cual valida la comparación de cantidades relativas de diferentes taxa procedentes de muestras arqueobotánicas de un mismo sitio. Sin embargo, en este trabajo no se realizaron correlaciones entre el número de fragmentos y el tamaño de los mismos por taxon, tal como proponen las autoras mencionadas, porque se considera que el submuestreo por curva de riqueza específica, inevitablemente necesario dada la alta cantidad de carbones recuperados, si bien permite representar con precisión variables que tienen que ver con la diversidad de taxa de la muestra total, no permite estimar fidedignamente la cantidad de carbones de cada taxón en la misma. Además, se sabe que si bien los patrones de fragmentación acontecidos durante la combustión pueden ser similares para cada taxón, es posible que dichos patrones se alteren durante los procesos posdepositacionales (Chrzavzez et al. 2011). Trabajos experimentales recientes demostraron que la fragmentación ocasionada, por ejemplo, por la compresión de sedimento, puede tener efectos variables en diferentes taxa, efectos que dependen también de las características de los procesos de combustión que les dieron origen (Chrzavzez et al. 2011). Estos efectos se pueden reflejar en una alteración del patrón de fragmentación original de cada taxón, dado por el proceso de combustión en sí mismo, que cambiaría la relación estipulada "mayor número de fragmentos-mayor peso" tanto a nivel de cada taxon individual como de la muestra en su conjunto (Chrzavzez et al. 2011). Por estos motivos es que para controlar el sesgo ocasionado por la fragmentación, y ante la imposibilidad de calcular la cantidad y tamaño de cada taxón para la totalidad de cada muestra, se prefirió en este caso realizar un análisis de correlación entre el número total de fragmentos de cada muestra y el peso correspondiente. Una correlación positiva y significativa entre estas variables permitiría constatar que ninguno de los niveles arqueológicos haya atravesado procesos posdepositacionales muy diferentes al resto (i.e niveles con elevado número de restos pero con bajo peso total, es decir compuestos por restos que en promedio serían de tamaño pequeño, podrían indicar la presencia de una fragmentación muy intensa; en tanto que niveles con escaso número de restos y un peso total elevado, es decir restos que en promedio serían grandes en tamaño, indicarían casi la ausencia de procesos posdepositacionales de fragmentación). Finalmente, el sesgo producido por la fragmentación debida a factores posrecuperación de los restos fue evaluado en términos de carbones con fracturas frescas.

\section{RESULTADOS}

Descripción anatómica de las muestras de referencia y clave identificatoria

Tal como se dijo anteriormente, la descripción correspondiente a las especies Adesmia pinifolia -tallo-, Adesmia obovata, Adesmia acuta, Baccharis neaei, Berberis empetrifolia -tallo y raíz-, Chuquiraga oppositifolia, Ephedra ochreata, Mulinun valentini, Schinus aff. roigii, y Senna arnottiana fue publicada con anterioridad por uno de nosotros (Andreoni 2010), por lo cual éstas no serán descriptas en el presente trabajo. Sí presentamos aquí la descripción anatómica de Ochetophila trinervis, Colliguaja intergerrima, Prosopis alpataco-tallo y raíz-, Adesmia pinifolia -raíz-, Anarthrophyllum sp. -tallo- y Lycium chilense -tallo- (Tabla 3). Dicha caracterización se realizó a partir de ejemplares de la colección propia de referencia, excepto aquella de las dos últimas especies, que fue realizada en base a fuentes bibliográficas: la primera fue descripta a partir de los trabajos de Cozzo (1950), Ancibor y Pérez de Micou (1995; 2002) y Marconetto (2002), mientras que la segunda en base al trabajo de Roig y Vidal (2006/2010). 


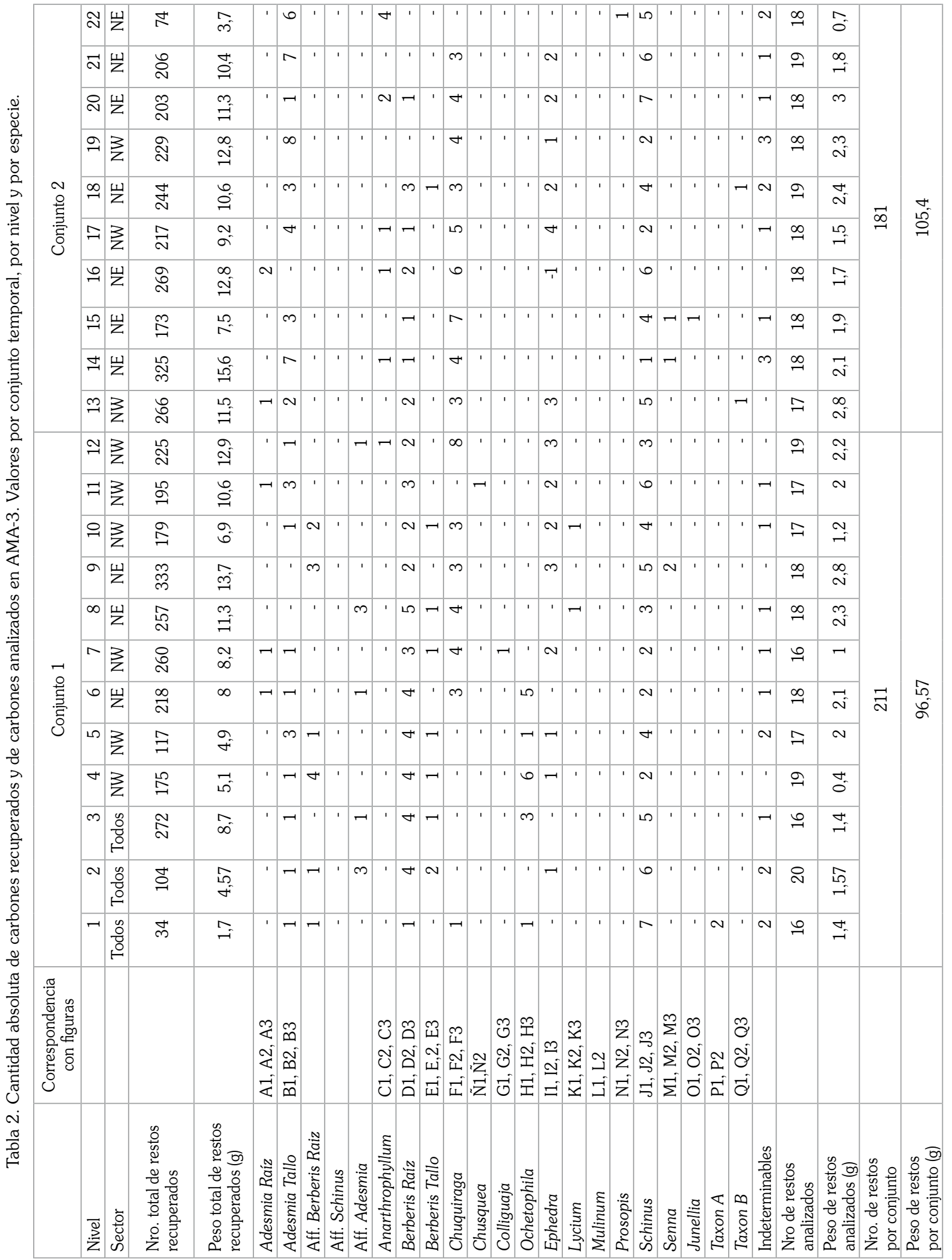




\begin{tabular}{|c|c|c|c|c|c|c|c|c|c|c|c|c|c|c|c|c|c|c|c|c|c|c|c|c|c|c|c|c|c|c|}
\hline & $\begin{array}{l}\bar{\pi} \\
0 \\
0\end{array}$ & & $\begin{array}{l}\circ \\
\text { 유 } \\
\text { }\end{array}$ & $\begin{array}{l}\hat{\sigma} \\
\stackrel{0}{\text { ลे }}\end{array}$ & $m$ & $\stackrel{\infty}{\infty}$ & $\Xi$ & 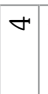 & 으 & $\Xi$ & ○ & $a$ & ๓ & -1 & $m$ & $\underline{-1}$ & 인 & $\sim$ & $\sim$ & -1 & $\stackrel{\circ}{\infty}$ & م & -1 & $\sim$ & $\sim$ & $\stackrel{\infty}{+}$ & 하 & \begin{tabular}{l}
$\hat{\sigma}$ \\
\multirow{\sigma}{*}{}
\end{tabular} & & \\
\hline \multirow{13}{*}{ 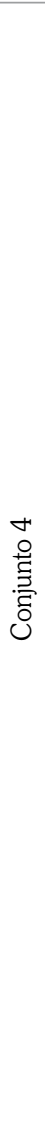 } & 웅 & z & \& & $\stackrel{\circ}{0}$ & ' & 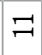 & ' & ' & 1 & ' & $\sim$ & I & I & I & I & 1 & - & 1 & I & ' & $N$ & ' & I & I & ' & $\sim$ & $\stackrel{\infty}{-1}$ & $\stackrel{0}{0}$ & \multirow{13}{*}{ ळి } & \multirow{13}{*}{ gे } \\
\hline & m & z & 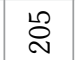 & $\stackrel{\sim}{\sim}$ & 1 & $\infty$ & 1 & 1 & 1 & -1 & 1 & I & 1 & I & 1 & 1 & 1 & 1 & 1 & I & 6 & ' & 1 & I & ' & $m$ & $\infty$ & 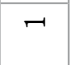 & & \\
\hline & $\infty$ & 㞱 & హ゙ & $\stackrel{+}{\sim}$ & 1 & $a$ & ' & ' & 1 & ' & ' & I & 1 & ' & I & 1 & 1 & ' & ' & ' & 0 & ' & ' & 1 & ' & $\sim$ & 드 & Oे & & \\
\hline & $\hat{n}$ & 之 & $\underset{\beth}{\Xi}$ & $\stackrel{N}{\tilde{n}}$ & 1 & 10 & 1 & I & 1 & 1 & I & I & I & ' & I & 1 & I & 1 & I & I & $\stackrel{\sim}{\mathcal{T}}$ & 1 & 1 & I & I & ' & 드 & Oे & & \\
\hline & లి & 㞱 & 옴 & $\overrightarrow{5}$ & 1 & $\exists$ & 1 & 1 & 1 & I & 1 & I & 1 & I & I & 1 & I & 1 & I & ' & ما & ' & 1 & 1 & I & ' & 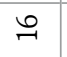 & $\stackrel{\sim}{\sim}$ & & \\
\hline & $\stackrel{\llcorner}{m}$ & z & $\stackrel{\Re}{\beth}$ & $\underset{\sigma}{v}$ & 1 & $m$ & ' & I & 1 & 1 & -1 & I & $\neg$ & ' & I & I & -1 & 1 & $\sim$ & ' & $a$ & 1 & 1 & I & ' & $\neg$ & $\stackrel{\infty}{\sim}$ & $\stackrel{\text { g }}{-}$ & & \\
\hline & ले & 之 & $\stackrel{\underset{\sim}{\sim}}{\sim}$ & $\stackrel{\infty}{\infty}$ & 1 & + & 1 & 1 & 1 & I & I & I & I & ' & I & 1 & -1 & 1 & 1 & ' & 익 & ' & 1 & 1 & ' & $\sim$ & $\Xi$ & $\stackrel{\infty}{0}$ & & \\
\hline & $m$ & z & $\stackrel{\infty}{\underset{\sim}{\sim}}$ & مL & 1 & $\infty$ & ' & $\sim$ & ' & ' & I & I & ' & I & I & 1 & -1 & ' & I & ' & $n$ & ' & ' & I & ' & ' & $\underset{\sim}{\infty}$ & $\underset{\leftarrow}{\stackrel{\sigma}{i}}$ & & \\
\hline & $\widetilde{m}$ & z & $\stackrel{\Xi}{\Xi}$ & $\stackrel{0}{\tilde{n}^{\prime}}$ & ' & 으 & ' & ' & ' & ' & $\sim$ & ' & 1 & ' & I & 1 & 1 & ' & ' & ' & 10 & ' & ' & I & ' & $\sim$ & 극 & $\approx$ & & \\
\hline & $\vec{m}$ & 之 & శెర & م & 1 & $\infty$ & 1 & $\sim$ & 1 & I & I & I & $m$ & I & I & 1 & -1 & 1 & I & ' & $m$ & 1 & 1 & 1 & 1 & 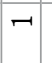 & $\infty$ & $\neg$ & & \\
\hline & ஓ & 之 & 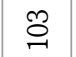 & $\begin{array}{l}\infty \\
\dot{m}\end{array}$ & 1 & $\sigma$ & 1 & ' & 1 & - & I & I & 1 & I & I & 1 & $m$ & 1 & I & ' & 윽 & - & 1 & 1 & 1 & - & ণ & $\stackrel{0}{2}$ & & \\
\hline & શ & 之 & 7 & $\begin{array}{l}\text { L } \\
\text { N }\end{array}$ & 1 & $\infty$ & I & ' & ' & I & I & I & $m$ & I & I & 1 & -1 & I & I & ' & $m$ & ' & I & 1 & 1 & - & 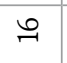 & $\underset{-}{\stackrel{\sigma}{\sigma}}$ & & \\
\hline & $\stackrel{\infty}{\sim}$ & 㞱 & ๙ & $\stackrel{\sim}{\sim}$ & 1 & م & $\sim$ & ' & I & 1 & 0 & I & 1 & I & I & I & ' & ' & I & ' & $\checkmark$ & ' & I & I & 1 & -1 & $\infty$ & $\hat{\sim}$ & & \\
\hline \multirow{5}{*}{ 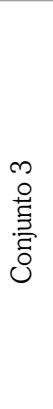 } & $\widehat{N}$ & z & ષ્ & $\overrightarrow{5}$ & $N$ & $\pi$ & 1 & I & I & ' & I & I & $m$ & 1 & I & ' & $m$ & 1 & 1 & ' & $\sim$ & I & 1 & I & 1 & - & $\stackrel{\infty}{\sim}$ & $\approx$ & \multirow{5}{*}{ হ } & \multirow{5}{*}{ N } \\
\hline & $\stackrel{\sim}{\sim}$ & 㞱 & ล̄ & $m$ & $\sim$ & $N$ & I & I & I & & I & 1 & $m$ & I & I & 1 & $m$ & 1 & 1 & ' & $\sim$ & I & I & I & 1 & - & $\infty$ & $\stackrel{\infty}{0}_{0}^{\infty}$ & & \\
\hline & $\stackrel{\llcorner}{\sim}$ & 㞱 & $\stackrel{\infty}{\underset{\sim}{+}}$ & бु & 1 & $\infty$ & I & I & I & - & 1 & I & -1 & I & I & 1 & $\sim$ & 1 & 1 & I & $m$ & I & I & I & 1 & $m$ & $\infty$ & $\infty$ & & \\
\hline & 岕 & 㞱 & $\stackrel{ }{~}$ & $\overbrace{}^{\infty}$ & $m$ & 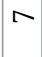 & ' & ' & -1 & -1 & ' & ' & -1 & ' & $\sim$ & ' & $\sim$ & I & ' & ' & ' & ' & ' & I & ' & $\sim$ & $\stackrel{2}{\sim}$ & $\approx$ & & \\
\hline & 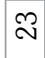 & 㞱 & $\underset{\sim}{ }$ & $\underset{\sim}{\sim}$ & 1 & $\sim$ & 1 & ' & ' & 1 & ' & I & $m$ & I & I & I & $\neg$ & I & I & . & ' & ' & ' & I & ' & I & 6 & $\stackrel{r}{\sigma}$ & & \\
\hline & $\underset{\stackrel{\rho}{c}}{\stackrel{\rho}{z}}$ & 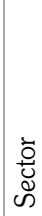 & 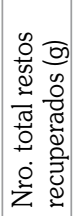 & 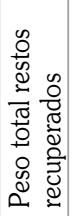 & 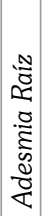 & 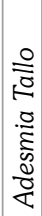 & 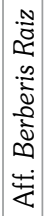 & 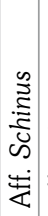 & 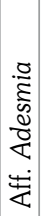 & 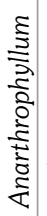 & 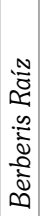 & 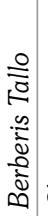 & 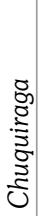 & 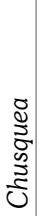 & 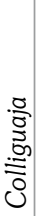 & 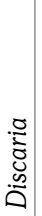 & $\begin{array}{l}\frac{0}{0} \\
\frac{0}{0} \\
\frac{2}{2}\end{array}$ & 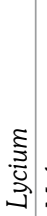 & 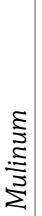 & \begin{tabular}{c}
0 \\
\hdashline \\
0 \\
0 \\
0 \\
0 \\
2
\end{tabular} & 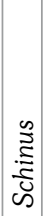 & 站 & $\begin{array}{l}\stackrel{g}{\bar{\Xi}} \\
\frac{5}{5}\end{array}$ & $\begin{array}{c}\varangle \\
\vdots \\
\vdots \\
0 \\
0 \\
\end{array}$ & 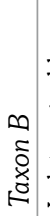 & 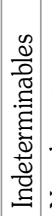 & 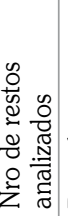 & 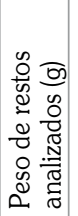 & 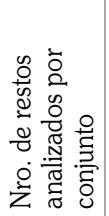 & 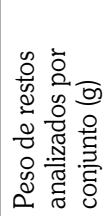 \\
\hline
\end{tabular}


Tabla 3. Principales caracteres diagnósticos de las especies identificadas en el presente trabajo y de aquellas que aún permanecen indeterminadas, tales como los taxa arqueológicos A y B.

\begin{tabular}{|c|c|c|c|c|c|c|c|c|c|c|}
\hline Rasgo según IAWA (1989) & $\begin{array}{l}\text { Adesmia } \\
\text { pinifolia } \\
\text { raíz }\end{array}$ & $\begin{array}{l}\text { Discaria } \\
\text { trinervis }\end{array}$ & $\begin{array}{l}\text { Prosopis } \\
\text { alpataco } \\
\text { tallo }\end{array}$ & $\begin{array}{l}\text { Prosopis } \\
\text { alpataco } \\
\quad \text { raíz }\end{array}$ & $\begin{array}{c}\text { Colliguaya } \\
\text { intergerrima }\end{array}$ & $\begin{array}{l}\text { Lycium } \\
\text { chilense }\end{array}$ & $\begin{array}{l}\text { Anartro- } \\
\text { phyllum } \\
\text { sp. }\end{array}$ & $\begin{array}{c}\text { Junellia } \\
\text { sp. }\end{array}$ & Taxon A & Taxon B \\
\hline Anillos de crecimiento demarcados & $\mathrm{X}$ & $\mathrm{X}$ & $\mathrm{X}$ & $\mathrm{X}$ & $\mathrm{X}$ & $\mathrm{X}$ & $\mathrm{X}$ & $\mathrm{X}$ & $\mathrm{X}$ & $\mathrm{X}$ \\
\hline Porosidad circular & $\mathrm{X}$ & & & & & $\mathrm{X}$ & & & & \\
\hline Porosidad semi-circular & & $\mathrm{X}$ & $\mathrm{X}$ & & $\mathrm{X}$ & & & $\mathrm{X}$ & $\mathrm{X}$ & \\
\hline Porosidad difusa & & & & $\mathrm{X}$ & & & $\mathrm{X}$ & & & $\mathrm{X}$ \\
\hline Vasos en bandas tangenciales & $\mathrm{X}$ & & & & $\mathrm{X}$ & & & $\mathrm{X}$ & $\mathrm{X}$ & $\mathrm{X}$ \\
\hline Vasos en patrón diagonal y/o radial & & & $\mathrm{X}$ & $\mathrm{X}$ & & & $\mathrm{X}$ & & & \\
\hline Vasos en patrón dendrítico & $\mathrm{X}$ & $\mathrm{X}$ & $\mathrm{X}$ & & & $\mathrm{X}$ & $\mathrm{X}$ & $\mathrm{X}$ & & \\
\hline $\begin{array}{l}\text { Vasos exclusivamente } \\
\text { solitarios ( } 90 \% \text { o más) }\end{array}$ & & & & & & $\mathrm{X}$ & $\mathrm{X}$ & $\mathrm{X}$ & $\mathrm{X}$ & \\
\hline Vasos agrupados frecuentes & $\mathrm{X}$ & $\mathrm{X}$ & & $\mathrm{X}$ & $\mathrm{X}$ & & & & & $\mathrm{X}$ \\
\hline $\begin{array}{l}\text { Vasos de dos clases diamétricas } \\
\text { distintas, en maderas de } \\
\text { porosidad no circular }\end{array}$ & & & $X$ & $X$ & & & & & & \\
\hline Vasos solitarios de contorno angular & & & & & & & & $\mathrm{X}$ & $\mathrm{X}$ & \\
\hline Fibras de pared muy delgada & $\mathrm{X}$ & & & & & & & $\mathrm{X}$ & $\mathrm{X}$ & $\mathrm{X}$ \\
\hline Fibras de pared delgada a gruesa & & & $\mathrm{X}$ & $\mathrm{X}$ & $\mathrm{X}$ & $\mathrm{X}$ & $\mathrm{X}$ & & & \\
\hline Fibras de pared muy gruesa & & $\mathrm{X}$ & & & & & & & & \\
\hline $\begin{array}{l}\text { Parénquima axial ausente o } \\
\text { extremadamente escaso }\end{array}$ & $\mathrm{X}$ & $\mathrm{X}$ & & & $\mathrm{X}$ & & & & $\mathrm{X}$ & $\mathrm{X}$ \\
\hline Parénquima axial difuso & & & & & & $\mathrm{X}$ & & & & \\
\hline $\begin{array}{l}\text { Parénquima axial } \\
\text { paratraqueal escaso }\end{array}$ & & $\mathrm{X}$ & & & & $\mathrm{X}$ & & & & \\
\hline $\begin{array}{l}\text { Parénquima axial } \\
\text { paratraqueal vasicéntrico }\end{array}$ & $\mathrm{X}$ & & $\mathrm{X}$ & $\mathrm{X}$ & & & & & & \\
\hline $\begin{array}{l}\text { Parénquima axial } \\
\text { paratraqueal aliforme }\end{array}$ & & & $X$ & & & & & & & \\
\hline $\begin{array}{l}\text { Parénquima axial } \\
\text { paratraqueal confluente }\end{array}$ & $\mathrm{X}$ & $X$ & $\mathrm{X}$ & $X$ & & & $X$ & & & \\
\hline $\begin{array}{l}\text { Parénquima axial marginal o } \\
\text { aparentemente en bandas marginales }\end{array}$ & $\mathrm{X}$ & & & & & & & & & \\
\hline Placa de perforación simple & $\mathrm{X}$ & $\mathrm{X}$ & $\mathrm{X}$ & $\mathrm{X}$ & $\mathrm{X}$ & $\mathrm{X}$ & $\mathrm{X}$ & $\mathrm{X}$ & $\mathrm{X}$ & $\mathrm{X}$ \\
\hline Punteaduras intervasculares opuestas & $\mathrm{X}$ & & & & & & & & & \\
\hline Punteaduras intervasculares alternas & $\mathrm{X}$ & $\mathrm{X}$ & $\mathrm{X}$ & $\mathrm{X}$ & $\mathrm{X}$ & $\mathrm{X}$ & $\mathrm{X}$ & $\mathrm{X}$ & $\mathrm{X}$ & $\mathrm{X}$ \\
\hline $\begin{array}{l}\text { Forma de las punteaduras } \\
\text { alternas poligonales }\end{array}$ & & & $\mathrm{X}$ & $X$ & $X$ & & & & & \\
\hline $\begin{array}{l}\text { Engrosamientos helicoidales } \\
\text { presentes en los elementos de vaso }\end{array}$ & $X$ & $\mathrm{X}$ & & & $X$ & $X$ & $X$ & $X$ & $X$ & $X$ \\
\hline $\begin{array}{l}\text { Engrosamientos helicoidales } \\
\text { a lo largo del cuerpo del } \\
\text { elemento de vaso }\end{array}$ & $X$ & $X$ & & & $X$ & $X$ & $X$ & & $X$ & $X$ \\
\hline $\begin{array}{l}\text { Traqueidas vasculares } \\
\text { vasicéntricas presentes }\end{array}$ & & $X$ & & $X$ & & $X$ & $X$ & & & $X$ \\
\hline $\begin{array}{l}\text { Fibras con punteaduras simples a } \\
\text { punteaduras con rebordes diminutos }\end{array}$ & $X$ & $X$ & $X$ & $X$ & $X$ & $X$ & $X$ & & $X$ & $X$ \\
\hline
\end{tabular}




\begin{tabular}{|c|c|c|c|c|c|c|c|c|c|c|}
\hline Rasgo según IAWA (1989) & $\begin{array}{c}\text { Adesmia } \\
\text { pinifolia } \\
\text { raíz }\end{array}$ & $\begin{array}{l}\text { Discaria } \\
\text { trinervis }\end{array}$ & $\begin{array}{l}\text { Prosopis } \\
\text { alpataco } \\
\quad \text { tallo }\end{array}$ & $\begin{array}{l}\text { Prosopis } \\
\text { alpataco } \\
\text { raíz }\end{array}$ & $\begin{array}{c}\text { Colliguaya } \\
\text { intergerrima }\end{array}$ & $\begin{array}{l}\text { Lycium } \\
\text { chilense }\end{array}$ & $\begin{array}{l}\text { Anartro- } \\
\text { phyllum } \\
\text { sp. }\end{array}$ & $\begin{array}{l}\text { Junellia } \\
\text { sp. }\end{array}$ & Taxon A & Taxon B \\
\hline $\begin{array}{l}\text { Fibras con punteaduras generalmente } \\
\text { en las paredes radiales o tangenciales }\end{array}$ & & $\mathrm{X}$ & & $\mathrm{X}$ & $\mathrm{X}$ & & & & & $\mathrm{X}$ \\
\hline $\begin{array}{l}\text { Espesamientos espiralados } \\
\text { en fibras del tejido básico }\end{array}$ & & & & & $\mathrm{X}$ & & & $\mathrm{X}$ & $\mathrm{X}$ & \\
\hline Fibras no-septadas presentes & $\mathrm{X}$ & $\mathrm{X}$ & $\mathrm{X}$ & $\mathrm{X}$ & $\mathrm{X}$ & $\mathrm{X}$ & & & & \\
\hline Células parenquimáticas fusiformes & $\mathrm{X}$ & & & $\mathrm{X}$ & & & $\mathrm{X}$ & & $\mathrm{X}$ & \\
\hline Dos células por serie parenquimática & & & $\mathrm{X}$ & & & $\mathrm{X}$ & & & & \\
\hline (3-4) células por serie parenquimática & $\mathrm{X}$ & & $\mathrm{X}$ & & & & & & & \\
\hline Radios exclusivamente uniseriados & & & & & $\mathrm{X}$ & $\mathrm{X}$ & & & & \\
\hline Radios $1-3$ seriados & $(1)-2-(1)$ & $1-(3)$ & $(1)-2-(3)$ & (3) & & & $(2)-(3)$ & $(1)-3$ & ¿? & $(1)-2-(3)$ \\
\hline $\begin{array}{l}\text { Radios grandes, comúnmente } \\
4-10 \text { seriados }\end{array}$ & $4-(5)$ & $4-(5)$ & & $(5)-6-(8)$ & & & (5)-7-(10) & & & \\
\hline Radios agregados & & $\mathrm{X}$ & & & & & & & & \\
\hline Altura de los radios mayor a $1 \mathrm{~mm}$ & $\mathrm{X}$ & $\mathrm{X}$ & & $\mathrm{X}$ & & $\mathrm{X}$ & & & & \\
\hline $\begin{array}{l}\text { Radios bajos estratificados, } \\
\text { altos no estratificados }\end{array}$ & $\mathrm{X}$ & & & & & & & & & \\
\hline $\begin{array}{l}\text { Parénquima axial y/o elementos } \\
\text { de vaso estratificados }\end{array}$ & $\mathrm{X}$ & & & & & & & & $\mathrm{X}$ & \\
\hline Fibras estratificadas & & & & $\mathrm{X}$ & & & & & & \\
\hline $\begin{array}{l}\text { Radios y/o elementos axiales } \\
\text { irregularmente estratificados }\end{array}$ & & & & & & & $\mathrm{X}$ & & & \\
\hline Cristales prismáticos presentes & & & $\mathrm{X}$ & $\mathrm{X}$ & & $\mathrm{X}$ & & & & \\
\hline $\begin{array}{l}\text { Cristales prismáticos en células } \\
\text { parenquimáticas axiales tabicadas }\end{array}$ & & & $\mathrm{X}$ & $\mathrm{X}$ & & & & & & \\
\hline Cristales prismáticos en fibras & & & $\mathrm{X}$ & $\mathrm{X}$ & & & & & & \\
\hline $\begin{array}{l}\text { Punteaduras vaso-radiales con } \\
\text { rebordes visibles; similares a } \\
\text { punteaduras intervasculares } \\
\text { en tamaño y forma a lo largo } \\
\text { de las células radiales }\end{array}$ & $\mathrm{X}$ & $\mathrm{X}$ & $\mathrm{X}$ & $\mathrm{X}$ & $\mathrm{X}$ & $\mathrm{X}$ & $\mathrm{X}$ & $\mathrm{X}$ & & $\mathrm{X}$ \\
\hline $\begin{array}{l}\text { Todas las células radiales } \\
\text { procumbentes }\end{array}$ & & $\mathrm{X}$ & $\mathrm{X}$ & & & & $\mathrm{X}$ & & & $\mathrm{X}$ \\
\hline $\begin{array}{l}\text { Todas las células del radio } \\
\text { erectas y/o cuadradas }\end{array}$ & & & & & $\mathrm{X}$ & $\mathrm{X}$ & & $\mathrm{X}$ & & \\
\hline $\begin{array}{l}\text { Células del cuerpo del radio } \\
\text { procumbentes con } 1 \text { hilera de células } \\
\text { marginales erectas y/o cuadradas }\end{array}$ & & $\mathrm{X}$ & & & & & $\mathrm{X}$ & $\mathrm{X}$ & & \\
\hline $\begin{array}{l}\text { Células del cuerpo del radio } \\
\text { procumbentes con } 2-4 \\
\text { hilera de células marginales } \\
\text { erectas y/o cuadradas }\end{array}$ & $\mathrm{X}$ & & & & & & & & & \\
\hline $\begin{array}{l}\text { Células del cuerpo del radio } \\
\text { procumbentes con más de } 4 \\
\text { hilera de células marginales } \\
\text { erectas y/o cuadradas }\end{array}$ & & $\mathrm{X}$ & & & & & & & & \\
\hline $\begin{array}{l}\text { Radios con células procumbentes, } \\
\text { cuadradas y erectas mezcladas }\end{array}$ & $\mathrm{X}$ & & & $\mathrm{X}$ & & & & & & \\
\hline $\begin{array}{l}\text { Cristales prismáticos en células } \\
\text { parenquimáticas axiales no tabicadas }\end{array}$ & & & & & & $\mathrm{X}$ & & & & \\
\hline
\end{tabular}


Se elaboró una clave dicotómica que reúne las especies descriptas en Andreoni (2010) con las descriptas en el presente trabajo, y que facilitó la diferenciación de los restos antracológicos:

\section{Clave de diferenciación de los géneros arqueológicos identificados}

0) estructura leñosa de monocotiledónea Chusquea

0 ') estructura leñosa de dicotiledónea

1) Cambium anómalo, vasos exclusivamente solitarios, punteaduras escalariformes --------------------Mulinun

1) Cambium normal-

2) Anillos de recorrido muy discontinuo alrededor del tronco, radios exclusivamente uniseriados

2') Anillos de recorrido continuo alrededor del tronco, radios uni o multiseriados

3) Vasos sin disposición dendrítica, parénquima axial raro o ausente-

4) Porosidad difusa

5) Parénquima vasicéntrico y confluente Vasos con espesamiento helicoidal, sin contenido celular en vasos, parénquima fusiforme y vasos estratificados - Adesmia raíz

6') Vasos con espesamientos reticulado, con contenido celular en vasos, gran cantidad de cristales en parénquima axial, sin estratificación

Prosopis

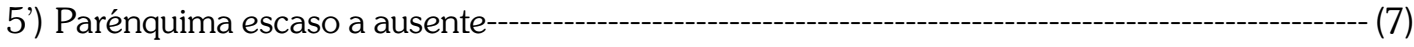

7) Vasos con disposición radial abundantes, espesamientos helicoidales, canales radiales en radios, placas de perforación simples-

7) Vasos sin disposición radial, la mayoría solitarios aunque algunos múltiples de 2 o 4 en disposición tangencial, traqueidas, vasos con placas de perforación foraminadas, campos de cruzamiento cupresoides

Ephedra

4) Porosidad semicircular, vasos en disposición tangencial en leño temprano --------------- (8)

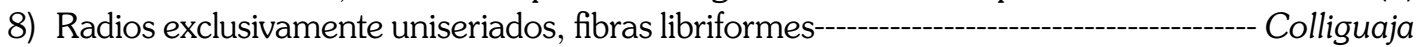

8') Radios uni a triseriados más comúnmente biseriados, fibrotraqueidas ----------------Chuquiraga

3) Vasos con disposición dendrítica

(9)

9) Parénquima paratraqueal confluente siguiendo el patrón dendrítico de los vasos, con o sin parénquima marginal, fibrotraqueidas muy escasas o ausentes------------------------------ (10)

10) Estratificación parcial o total

11) Radios y/o elementos axiales irregularmente estratificados------------------Anarthrophyllum

11') Estructura estratificada en vasos y parénquima, punteaduras opuestas y escalariformes, vasos agrupados presentes Adesmia tallo

10') Sin estructura estratificada en parénquima y vasos, punteaduras alternas, vasos agrupados ausentes, con espesamientos espiralados en los vasos Senna

9) Parénquima apotraqueal o escaso o ausente, fibrotraqueidas abundantes (12)

12) Parénquima apotraqueal, no se distingue disposición ulmoide en leño temprano, brazos del patrón dendrítico comúnmente gruesos, Ochetophila

12') Parénquima escaso o ausente, disposición ulmoide del leño temprano evidente, brazos del patrón dendrítico finos 


\section{LOS TAXA ARQUEOLÓGICOS IDENTIFICADOS Y SU REPRESENTACIÓN EN EL SITIO AMA-3}

El total de restos antracológicos recuperados en el sector norte de AMA-3 es de 6.650. De dicho total se analizaron 701 carbones de madera (Tabla 2), de los cuales se identificó el 92,29\% que representa un total de 647 carbones. La muestra posee representantes de 15 taxa vegetales distintos (Fig. 4 A-B) que se distinguen en términos de su cantidad relativa \% (Fig. 4 A): Adesmia (27,53\%)-tallo (25,68\%) y raíz, (1,85\%)-, Schinus (25,68\%), Chuquiraga $(11,84 \%)$, Berberis $(9,84 \%)$-tallo $(1,28 \%)$ y raíz (8,56\%)-, Ephedra (7,13\%), Ochetophila (2,28\%), Anarthrophyllum (2\%), Senna (0,71\%), Colliguaja (0,43\%), Mulinun (0,29\%), Chusquea (0,14\%), Junellia $(0,14 \%)$, y Prosopis $(0,14 \%)$ (posiblemente Prosopis alpataco, por su similitud con los rasgos anatómicos de la muestra de referencia y por ser, además, una especie comúnmente presente en la cuña patagónica de la provincia mendocina).

De la muestra identificada, unos pocos ejemplares debieron ser definidos como afines a determinados taxa, ya que el grado de vitrificación de sus tejidos en el plano transversal dificultó el reconocimiento total de caracteres diagnósticos en dicho plano (Fig. 5), debiéndose utilizar principal-

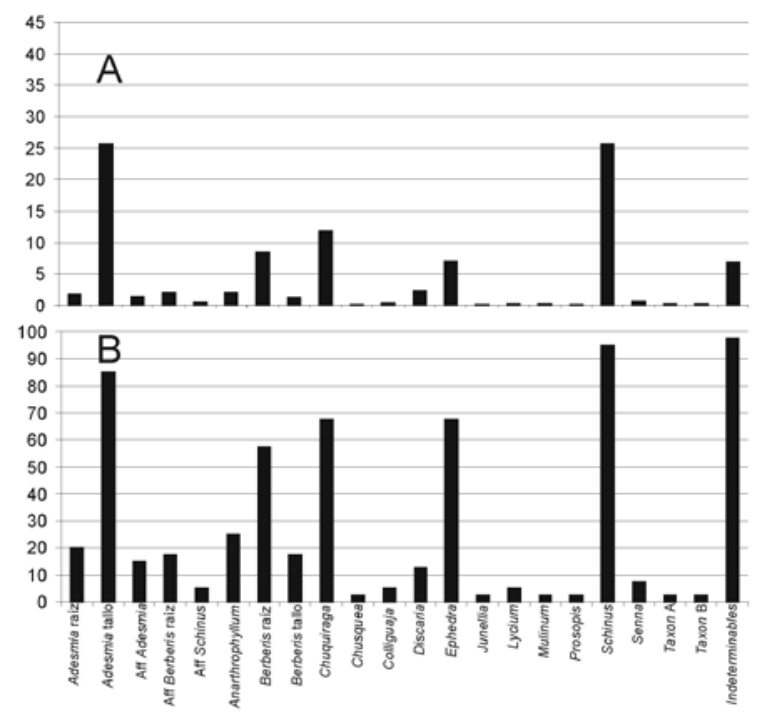

Fig 4. Taxa representados en la secuencia completa del sitio AMA-3. A. Cantidad relativa \% (en base al conteo absoluto). B. Ubicuidad. mente caracteres de los CLT y CLR. Tal es el caso de: aff. Adesmia tallo (1,43\%), aff. Schinus (0,57\%), y aff. Berberis raíz (2\%).

El análisis de correlación entre el número de fragmentos efectivamente recuperados por nivel arqueológico y el peso de los mismos dio como resultado para un $\mathrm{n}=40$ niveles, un índice de correlación de 0,91 y un nivel de significación $<0,01$ (Fig. 7). Esta asociación entre el número de fragmentos y el peso total de los restos de cada nivel permite estimar que ningún nivel arqueológico atravesó procesos posdepositacionales muy diferentes al resto (i.e. no se observaron niveles con elevado número de restos pero con bajo peso total, lo que podría indicar la presencia de una fragmentación muy intensa; ni niveles con escaso número de restos y un peso total elevado, lo que indicaría muy poca fragmentación). Asimismo, la fragmentación debida a factores posrecuperación de los restos y evaluada en términos de carbones con fracturas frescas, ha sido prácticamente nula en el sitio AMA-3.

El análisis por conjunto temporal (Fig. 8 y 9) permitió el reconocimiento de algunas prácticas de uso de leña (i.e. selección, obtención, temperaturas de combustión) para cada lapso específico y comparar unos con otros permitió establecer similitudes y diferencias a lo largo de toda la secuencia cronológica del sitio arqueológico AMA-3. En el caso del Conjunto 4, momentos más antiguos del sitio, correspondiente al lapso temporal que va de los 8.900 a los 7.600 años AP (Tabla 1), se analizaron 230 carbones, de los cuales pudieron identificarse el 93,04\%. Los taxa representados en mayor cantidad fueron: tallo de Adesmia (40,87\%) y de Schinus

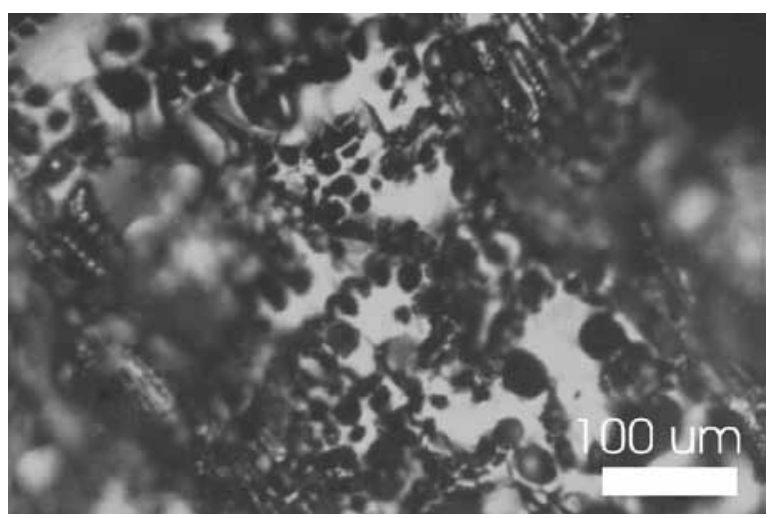

Fig 5. Ejemplares de aspecto vítreo: corte transversal de Aff raíz de Berberis. 

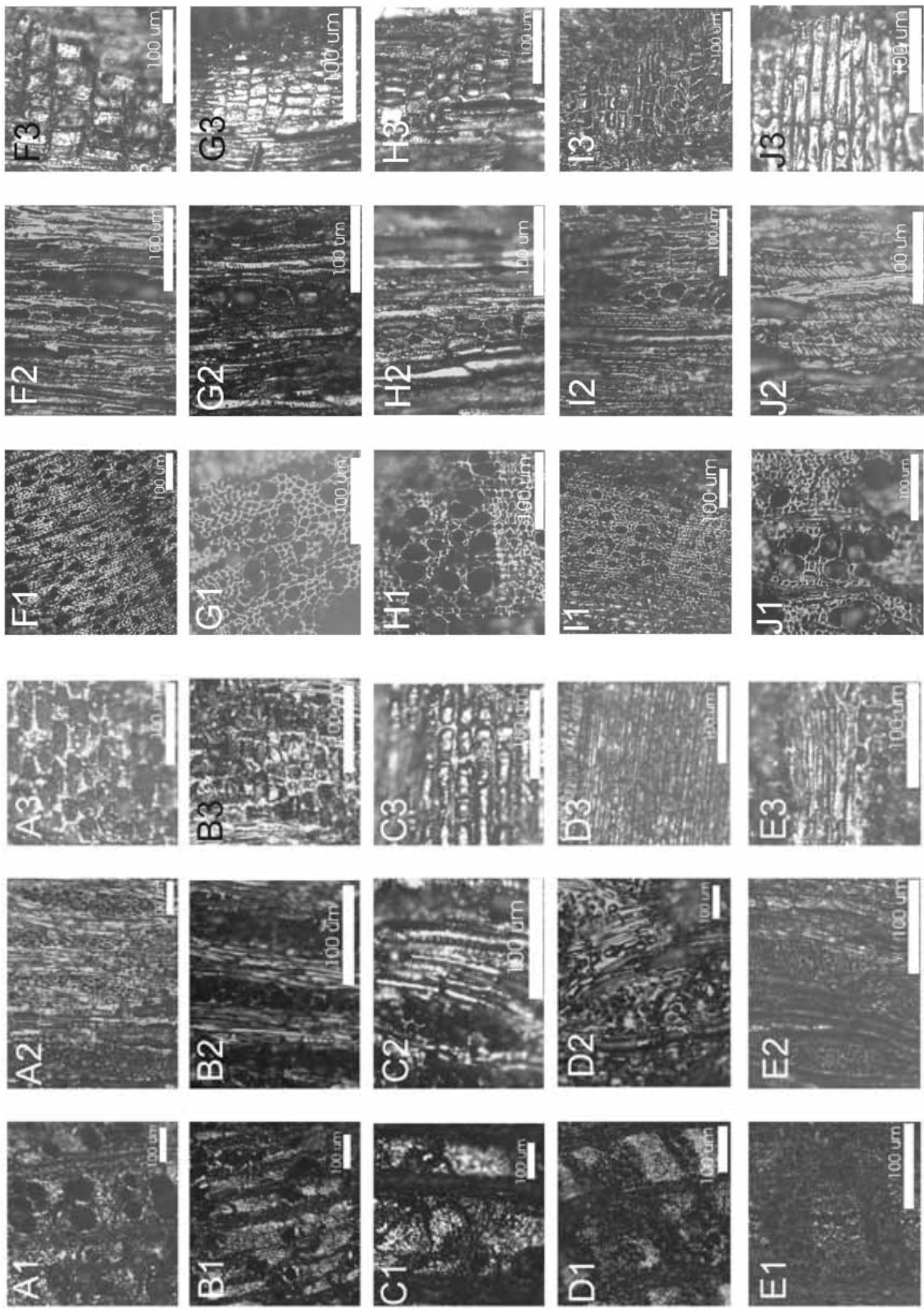

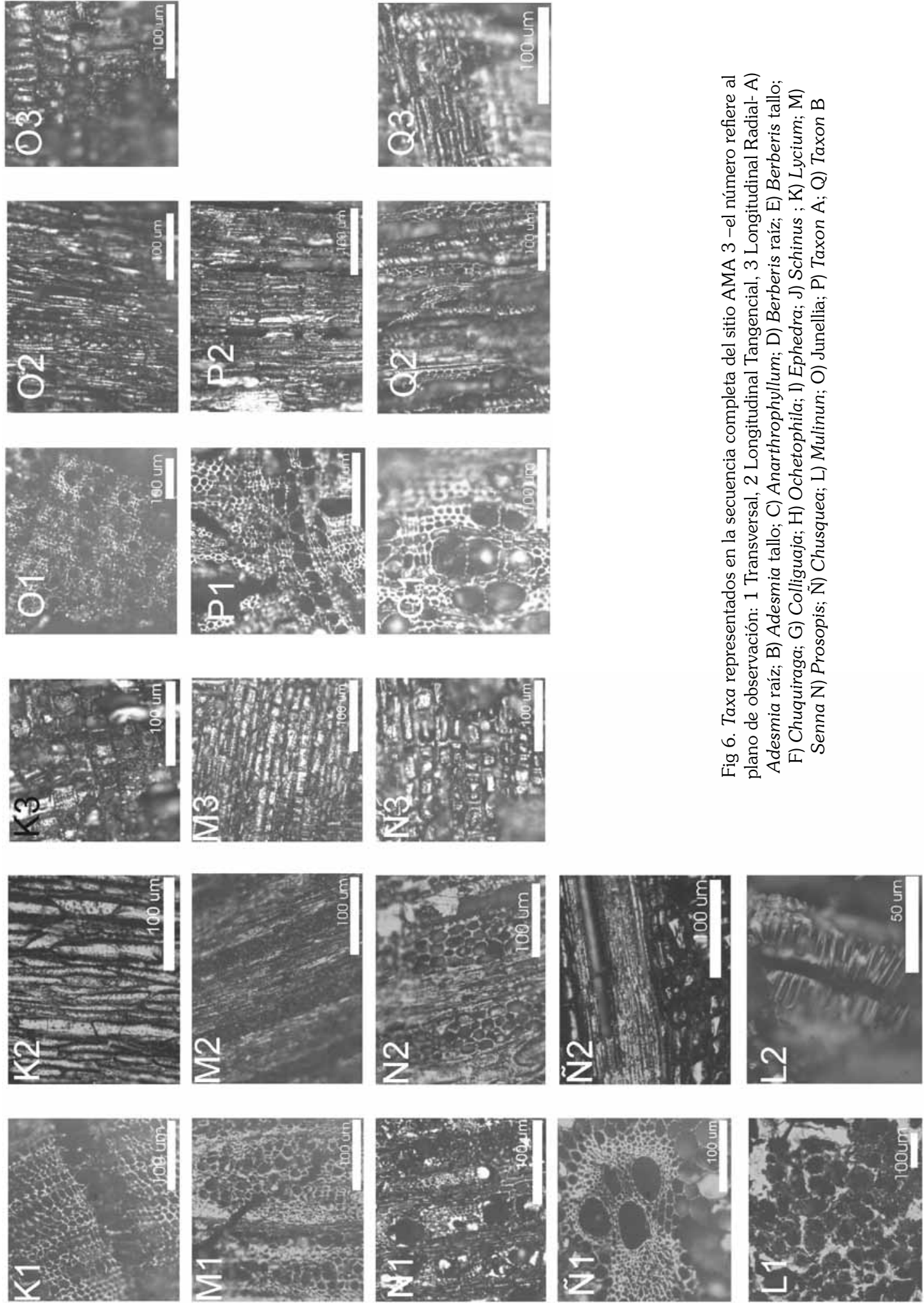


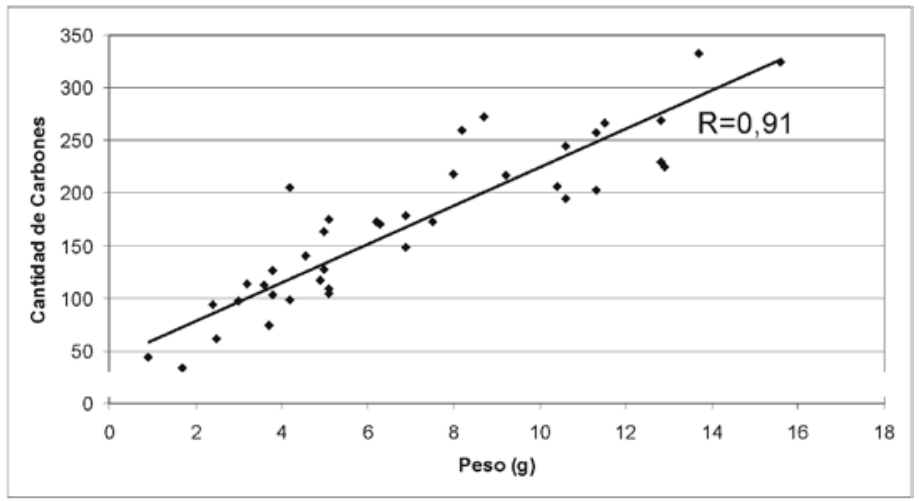

Fig. 7. Correlación entre cantidad y peso de carbón de madera recuperado por nivel arqueológico.

(35,65\%), en menor medida se identificaron Berberis raíz (4,78\%), Ephedra (3,91\%), Chuquiraga (3,04\%), y aff. Schinus (1,74\%). Otros géneros presentaron porcentajes menores al $1 \%$, por ejemplo aff Berberis raíz, Anarthrophyllum y Mulinun (0,87\%) y Senna (0,43\%) (Fig. 8). Adesmia y Schinus estuvieron en todas las muestras analizadas del conjunto, por lo que presentaron una ubicuidad del 100\%, mientras que los restantes géneros presentan valores inferiores al 50\% (Fig. 9). El Conjunto 3, que abarca un lapso temporal que va desde los 7600 a los 5000 años AP (Tabla 1), se encuentra representado por 79 carbones de los cuales fue posible identificar el 91,14\%. En éste se reconoció la presencia de 6 géneros. El representado en mayor cantidad fue Adesmia $(48,1 \%)$-tallo $(39,24 \%)$ y raíz $(8,86 \%)$-, seguido por Ephedra (13,92\%), Chuquiraga (13,92\%), Schinus (8,86\%), Colliguaja (2,53\%), Anarthrophyllum $(2,53 \%)$ y los carbones identificados como aff. a Adesmia (1,27\%) (Fig. 8). La ubicuidad calculada para el Conjunto 3 (Fig. 9) muestra a Adesmia tallo, Chuquiraga, y Ephedra, como los géneros con los valores más elevados (100\%), mientras que se aprecia una marcada disminución en Schinus (60\%), en relación al Conjunto 4 . Un elemento relevante del Conjunto 3 es la incorporación al registro de la raíz de Adesmia, que presentó valores relativamente altos en cantidad, y un porcentaje de ubicuidad (60\%) igual al de Schinus. En relación al Conjunto 2, que abarca un lapso temporal que va desde los 5.000 a los 2.200 años AP, se analizó un total de 181 carbones, de los cuales fue posible identificar el 90,61\% de los mismos. Se identificó la presencia de 8 géneros, de los cuales el más representado fue Adesmia (24,31\%)-tallo (22,65\%) y raíz (1,66\%), seguido por Chuquiraga (21,55\%), Schinus (23,20\%), Ephedra (8,29\%), y, en menor medida, Berberis $(6,63 \%)$-tallo $(0,55 \%)$ y raíz (6,08\%)- y Anarthrophyllum (4,97\%). Porcentajes menores aún, presentaron Senna (1,10\%), Prosopis (0,55\%), Junellia $(0,55 \%)$ y Taxon B (1,10\%). Los porcentajes de ubicuidad obtenidos para el Conjunto 2 se aprecian en la Fig 9. Este conjunto muestra un aumento en la representación de Schinus (100\%) con respecto al conjunto anterior, que iguala los valores del Conjunto 4 y cuya ubicuidad se va a mantener luego en el Conjunto 1 (ver más adelante). El Conjunto 2 presenta también altos valores de ubicuidad para Adesmia y Chuquiraga (90\% cada una), seguidos por Berberis raíz y Ephedra (70\% cada uno), y por Anarthrophyllum (50\%). Por último, del Conjunto $\mathbf{1}$, que cronológicamente abarca los últimos 2200 años AP, se analizaron 211 carbones, de los cuales se identificó el 93,37\%. Los taxa con mayor representatividad en términos de cantidad absoluta fueron Schinus (23,22\%) y Berberis (21,8\%)-tallo (3,79\%) y raíz (18,01\%)-. A esta última categoría podemos sumar la de aff. Berberis raíz (5,69\%), lo cual sumaria un total de $26,77 \%$ para la raíz de Berberis, cifra que podría estar relacionada con una importancia creciente de las raíces de dicho género como combustible durante este periodo. A estos valores le siguen aquellos de Chuquiraga (12,32\%), Adesmia $(8,06 \%)$-tallo $(6,64 \%)$ y raíz (1,42\%)- y aff. Adesmia (4,27\%), Ochetophila (7,58\%), Ephedra (7,11\%). En menor medida se presentaron Senna (0,95\%), Taxon A (0,95\%), Colliguaja (0,47\%) y Chusquea $(0,47 \%)$ (Fig. 8). Con respecto a los valores de ubicuidad (Fig. 9), el Conjunto 1 nos muestra una continuidad con el conjunto anterior, que se observa por un predominio 


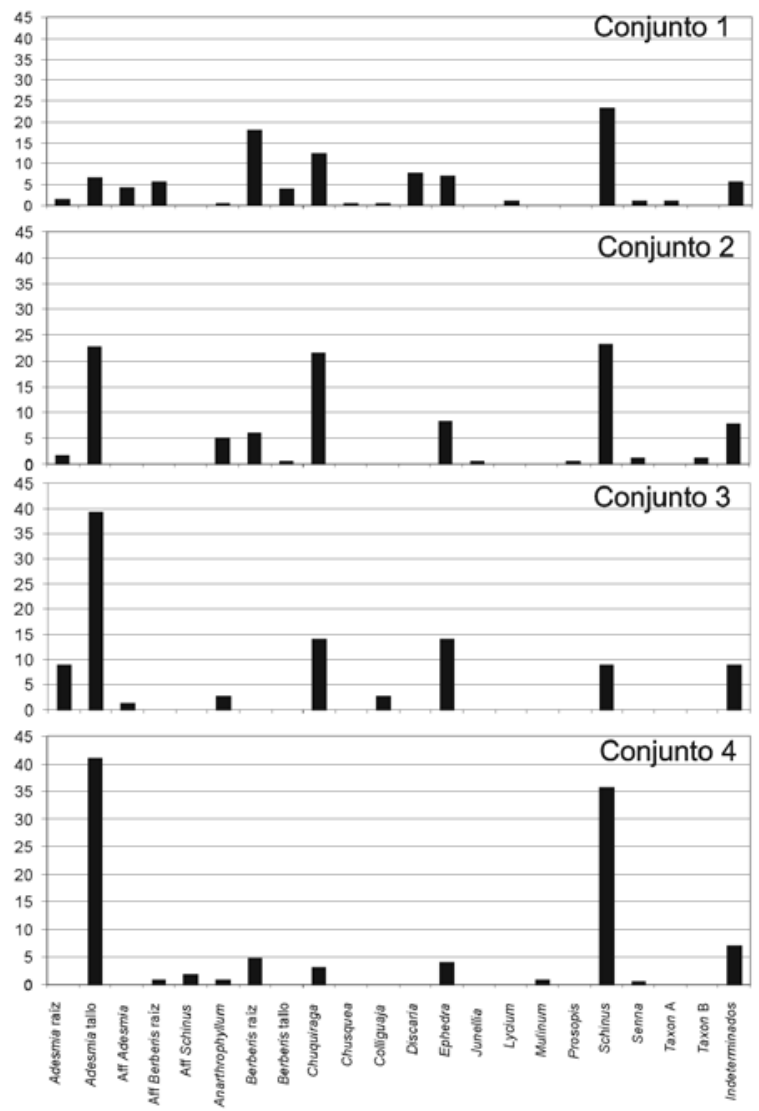

Fig 8. Cantidad relativa \% (en base al conteo absoluto) de los taxa en cada conjunto temporal del sitio arqueológico AMA-3.

de Schinus y de raíz de Berberis (100\% de ubicuidad cada uno), seguidos por Adesmia (83,3\%), Ephedra $(67,7 \%)$ y el tallo de Berberis (58,3\%).

\section{DISCUSIÓN}

Los resultados obtenidos a partir del registro antracológico de AMA-3, considerados conjuntamente con los distintos escenarios paleoambientales que se fueron sucediendo a lo largo del Holoceno, las propiedades físicas de la madera, así como la información procedente de fuentes escritas y los usos dados por poblaciones actuales a los distintos taxa, nos permiten evaluar algunas tendencias en cuanto a la explotación del recurso leñoso en el sitio.

Considerando el sitio arqueológico en su conjunto, se observa una diversidad importante de taxa $(n=15)$ representados en la secuencia temporal del mismo. A pesar de dicha diversidad, hay una

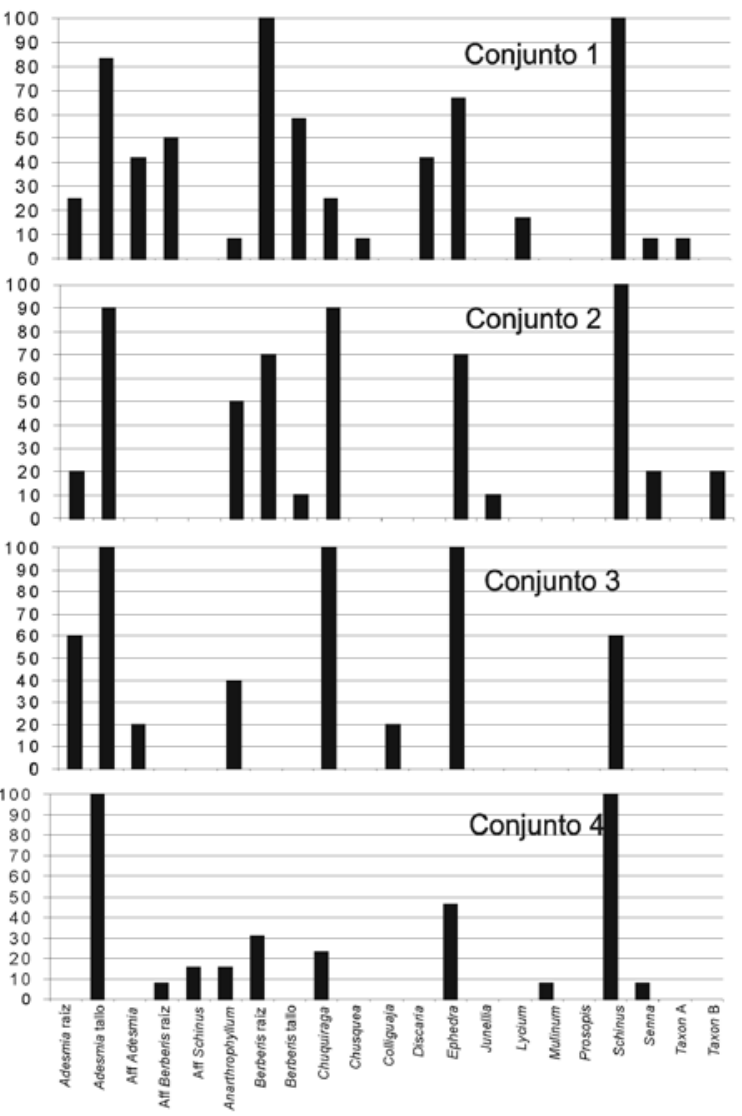

Fig 9. Porcentaje de ubicuidad de cada taxón en cada conjunto temporal del sitio arqueológico AMA 3.

preponderancia en términos de cantidad relativa de restos antracológicos de dos géneros principales: Adesmia y Schinus, a los que le siguen en orden de importancia Ephedra, Chuquiraga y Berberis. Se observa también una correspondencia entre los valores de cantidad relativa y ubicuidad, siendo los taxa más numerosos aquellos que poseen mayores valores de ubicuidad, es decir, que se hallan presentes en mayor cantidad de niveles arqueológicos de los cuatro conjuntos temporales considerados. Los taxa utilizados se corresponden con aquellos nativos y disponibles en el área tanto en la actualidad como en el pasado (excepto en el caso de Chusquea, ver más abajo).

Se observa que, si bien es interesante que el registro arqueobotánico de AMA-3 presenta solo elementos Andino-Patagónicos a lo largo de toda su secuencia temporal (a excepción de un solo ejemplar de Prosopis), hay algunos géneros registrados en las 
transectas modernas y en los registros polínicos que están ausentes en el registro arqueobotánico (i.e. Senecio, Fabiana, entre otros). También existen diferencias en cuanto a la abundancia relativa de ejemplares de cada taxa recuperado y su abundancia moderna en la región (por ejemplo, Anarthrophyllum, que según Hernández (1996) debería haber sido uno de los taxa más utilizados por su disponibilidad y calidad combustible, se encuentra en escasa cantidad en el registro arqueológico. Tal como se dijo anteriormente, se sabe que la representatividad diferencial del los taxa en el registro arqueológico está condicionada, además de por las prácticas culturales pasadas de uso de leña, por patrones que tienen que ver con respuestas diferenciales al proceso de carbonización, con procesos posdeposicionales (fragmentación, migración), y con factores posrecuperación de los restos (Chabal 1988, 1990, 1992; Théry-Parisot et al. 2010, Piqué i Huerta 1999). Ya se mencionó que a pesar de las limitaciones para caracterizar patrones de fragmentación por taxon producidos durante la combustión, se pudo comprobar un comportamiento aparentemente similar de los procesos posdepositacionales de fragmentación para los distintos niveles arqueológicos del sitio AMA-3, demostrado por el alto índice de correlación entre número de fragmentos y peso de cada muestra completa. Esto, sumado a que la fragmentación debida a factores posrecuperación de los restos ha sido prácticamente nula, nos permitiría interpretar las diferencias cuantitativas observadas en los conjuntos comparados como derivadas de prácticas culturales diferentes en lo que respecta a la selección y uso de materiales combustibles. Sin embargo, existen otras variables intrínsecas a la madera que inciden en la representatividad diferencial de los taxa, por ejemplo, la densidad. En este caso, los taxa identificados en AMA-3 poseen valores similares de densidad que van de 0,46 a 0,58, excepto en el caso de Mulinum, que posee un valor de 0,24 (ver Andreoni 2010). Por tal motivo se puede pensar que salvo Mulinum, que podría estar algo subrepresentado en el sitio por su baja densidad y alta posibilidad de transformarse en cenizas, el resto de los taxa presentes tienen similar potencial de preservación a iguales condiciones de carbonización. En este sentido, y volviendo al caso de Anarthrophyllum, madera de características semi-duras, su baja frecuencia registrada podría corresponderse con una baja tasa de utilización.
Hacia finales del Holoceno Temprano se observa, en el registro arqueológico del sitio AMA-3 la presencia de roedores como Andalgalomys, que permite correlacionar el comienzo de la secuencia de ocupación con un periodo climático más cálido que el anterior. Durante este momento se ha propuesto que este alero formó parte de un sistema mayor de asentamientos, dentro del cual fue utilizado como campamento de actividades específicas (Neme 2002). En este periodo la vegetación circundante al alero debió ser de tipo Andino-Patagónica, posiblemente con elementos Puneños, según se observa en el registro polínico (Páez et al. 2010). El registro antracológico en este periodo (conjunto 4), si bien registra una riqueza de 9 taxa, se caracteriza por un predominio muy marcado de tallos de Adesmia y Schinus, no solo, en cuanto a su cantidad relativa $\%$, sino también en su ubicuidad. Es interesante destacar que en la actualidad tanto la madera de Adesmia como la de Schinus son muy apreciadas como combustible (Hernández 2002; Ruiz Leal 1972), aunque Adesmia es más preferida sobre todo por la duración de sus brasas. Si bien Adesmia y Schinus seguramente han estado ampliamente disponibles en los alrededores del sitio, es evidente que estas leñas han sido seleccionadas por las sociedades pasadas de entre otras especies distribuidas en la zona y también abundantes como pueden haber sido Anarthrophyllum, Senna, Mulinum y Chuquiraga. Entre los ejemplares de Schinus de los niveles 28 al 38 se observan aquí especímenes con y sin aperturas radiales. Según trabajos experimentales realizados por nosotros anteriormente (Andreoni et al. 2010), sabemos que estas aperturas tienen que ver con diferencias en las temperaturas de combustión. Es conocido que dichas temperaturas afectan las características morfológicas de las maderas (Braadbaart y Poolel 2008). Se ha observado que especímenes de Schinus carbonizados a altas temperaturas $\left(700^{\circ} \mathrm{C}\right)$ desarrollan aperturas en el parénquima radial axial, mientras que aquellos carbonizados a bajas temperaturas $\left(400^{\circ}\right)$ no las desarrollan (Andreoni et al. 2010). Por tal motivo se piensa que los carbones de este nivel pudieron provenir de fuegos que tuvieron distintas características y funciones. La marcada concentración en el uso de las leñas más preciadas para la zona y la evidencia de fuegos de altas temperaturas podrían estar relacionadas con una mayor necesidad de 
fuegos para calentamiento, en un período que fue más frío que los posteriores, o con la presencia de ocupaciones estacionales invernales, o bien con el desarrollo de actividades específicas para las cuales se pudo haber requerido de la producción de fuegos con elevada temperaturas. Por ejemplo, dado que el registro faunístico del conjunto observa la preponderancia de restos de guanaco, en detrimento de animales más pequeños (ver Neme 2007), estos fuegos pueden haber servido para la cocción de piezas grandes de animales.

Posteriormente, durante el Holoceno Medio (8.000-4.000 AP), y aunque aún existen hiatus estratigráficos en varias de las secuencias polínicas de la región, se ha propuesto que se habría producido un paulatino proceso de desertificación (Páez et al. 2010; Zárate 2002), que hasta el momento no pudo ser corroborado con los registros polínicos disponibles (Páez et al. 2010). No obstante ello, se acepta que la composición florística de afinidad Andino-Patagónica y de Monte habría sido resistente al paulatino incremento de la temperatura y a la consecuente modificación del los sistemas fluviales (Páez et al. 2010). Arqueológicamente se ha propuesto que durante el Holoceno Medio, las poblaciones humanas se habrían asentado principalmente a lo largo de los valles fluviales, los cuales debieron funcionar como refugio ante dichas condiciones de mayor aridez (Gil et al. 2005). Recientemente esta disminución en la visibilidad arqueológica, durante el Holoceno medio, se ha relacionado con una mayor movilidad de las poblaciones humanas que ocuparon la región (Neme y Gil 2009), aunque en la actualidad este tópico sigue siendo tema de debate e investigación (García 2010; Neme y Gil 2010). El registro antracológico correspondiente a este periodo (conjunto 3), muestra una marcada disminución en la riqueza de taxa $(n=6)$. Si bien existe aún una preponderancia en el uso del tallo de Adesmia, su cantidad relativa disminuye con respecto al periodo anterior. Además, se incorpora al registro el uso de raíces de Adesmia. El mismo, podría deberse a una mayor disponibilidad de la parte subterránea de esta planta por la ocurrencia de los periodos de neo-glaciación acaecidos en la región hacia los 5.700 años AP (Espizúa 2000 en Gil et al. 2005). Está comprobado que el efecto de una acumulación inusual de nieve sobre las plantas de Adesmia puede quebrar y arrancar las matas de raíz, tal como lo observaron Ruiz Leal y Roig (1955), a diferencia de las plantas de Schinus y Berberis, que no se ven mayormente afectadas. De esta manera, la incorporación de las raíces de Adesmia en el sitio podría relacionarse con este evento de neo-glaciación. Otra posible interpretación de la incorporación de raíz de Adesmia es que ésta se relacione con una mayor intensificación en el uso de este género, dada por una ocupación más intensa del valle. Asimismo, durante este conjunto 3 aumentan las cantidades relativas y la ubicuidad de Chuquiraga y Ephedra. Sabemos que las especies de Chuquiraga son buenas para iniciar el fuego, incluso su nombre vernáculo "ardegras" o "monte chirriador" deja ver su capacidad de fácil combustión. La información etnobotánica señala que las matas de Chuquiraga son utilizadas para prender fuegos para hacer señales a largas distancias tanto en Patagonia (Ancibor y Pérez de Micou 2002), como por las poblaciones rurales del sur de Mendoza, sin necesidad siquiera de desplantarlas. Su alta ubicuidad a lo largo del conjunto 3 (e incluso posteriormente en el 2) podría deberse a una ocupación intermitente del sitio, por lo cual su presencia estaría indicando un mayor número de encendido de fuegos, o la realización de combustiones más expeditivas. Con respecto a Ephedra, no contamos con información etnobotánica, ni actualística en cuanto a su uso como combustible, pero sabemos que su densidad la ubica dentro de la categoría de maderas semi-duras junto con Adesmia y Schinus y que regionalmente se presentan algunos ejemplares arbustivos que alcanzan hasta los dos metros de alto como Ephedra boelckei. Esto nos permite pensar en que sus propiedades debieron ser conocidas por las poblaciones locales que habitaron AMA-3 y que ésta pudo ser un buen sustituto de Adesmia. Resulta llamativa, durante este periodo, la reducción en cantidad relativa (no en ubicuidad) del género Schinus, en relación al conjunto 4 . La mencionada disminución en el registro no se correlacionaría con cambios ambientales ya que, como se dijo anteriormente, los registros polínicos avalan que la vegetación existente habría resistido la creciente desertificación ocurrida durante el Holoceno Medio. Esta disminución en la cantidad y ubicuidad de Schinus pudo deberse o bien a una disminución en su utilización, o, más probablemente a un sesgo relacionado con las técnicas de muestreo implementadas, previo al desarrollo de estos estudios, para realizar las dataciones $\mathrm{C} 14$ del sitio. Prácticamente 
todos los carbones del nivel 23 fueron utilizados para realizar fechados radiocarbónicos sin identificación taxonómica previa. Schinus es el taxon que posee los valores más grandes de tamaño de los carbones recuperados, por lo cual es probable que haya constituido una porción importante de la muestra para datar, por lo que el uso de Schinus durante el conjunto 3 pudo ser mayor que el registrado en este trabajo. Desafortunadamente, la cuadrícula contigua (A-1) carece de evidencia de ocupación durante el Holoceno Medio (Dieguez y Neme 2003), como para ampliar la muestra de este periodo y contrastar la hipótesis planteada.

La ocupación del alero durante el Holoceno Tardío (conjuntos 2 y 1) corresponde al momento a partir del cual se establecen las condiciones climáticas actuales (Páez et al. 2010). Durante estos conjuntos la riqueza de taxa aumenta a 10 y 12 respectivamente. Ya no se observa preponderancia de uso de uno o dos taxa sino que las cantidades relativas de cada taxon en particular disminuyen, al tiempo que aumenta el número de taxa utilizados. Por ejemplo, Adesmia, presenta una disminución progresiva en el uso del tallo desde el conjunto 4 al 1, al tiempo que se registra el uso de raíces desde el conjunto 3 , que continúa en el 2 y 1 . El uso de Berberis tallo aparece recién en el conjunto 2 y persiste en el 1 . Aunque aquí su uso fue de carácter combustible, cabe aclarar que durante el Holoceno tardío la madera de Berberis comienza a utilizarse para la confección de artefactos en otras partes de Patagonia. Por ejemplo, B. illicifolia se reservaba para la confección de mangos y astiles por los habitantes originarios de Tierra del Fuego (Gusinde 1937 en Pique i Huerta 1999), y para la confección de arpones en la Costa Norte de Santa Cruz (sitio arqueológico Cueva del Negro, (Capparelli et al. 2009). Por otro lado, el uso de las raíces de este género se incrementa significativamente en los conjuntos 2 y 1 de AMA-3. Las raíces de Berberis alcanzan mayor grosor que su madera y son de muy buena calidad como combustible según información proporcionada por distintas fuentes (Ancibor y Pérez de Micou 2002; Andreoni 2010), lo cual fue demostrado experimentalmente por March (1992).

Es de destacar que otros taxa como Ochetophila, Lycium, Junellia, Taxon A y B, recién aparecen también en estos conjuntos, estando ausentes en los anteriores. Con respecto a Ochetophila, denomi- nada comúnmente como "chacay", los estudios de Agüero Blanch (1971), que detallan el proceso de elaboración y cocción de alfarería, permiten conocer que las poblaciones rurales de Malargüe lo usaban tradicionalmente, junto al algarrobo (Prosopis) y al molle (Schinus), para la cocción de las piezas cerámicas. En nuestra área de estudio este género se encuentra representado por Ochetophila trinervis (chacay), un árbol cuya altura puede alcanzar hasta 5 metros o más, y que se encuentra siempre asociado a los cursos de agua y restringido en cuanto a su distribución. Por información actual sabemos que su madera solo se colecta una vez que la crecida ha producido la ruptura natural de sus ramas (comunicación personal Dra Gabriela Díaz). Es llamativo que siendo prácticamente el único árbol en la región, su madera haya sido utilizada como combustible solo en los últimos periodos de ocupación del alero, incluso su presencia se registra en los niveles más tardíos del sitio (Tabla 2) y es un elemento poco ubicuo en el conjunto 1 y en el sitio en general (Fig. 4 y 9). Posiblemente su madera fuera destinada a otros usos y solo fue explotada como combustible cuando disminuyó la disponibilidad de otras especies.

En suma, durante el conjunto 1, no hay preponderancia de uno o dos taxa sino que son varios los que presentan mayor cantidad relativa, que, en orden de importancia, corresponden a Schinus, raíz de Berberis, Chuquiraga, Ochetophila, Ephedra, tallos y raíces de Adesmia, y tallos de Berberis; todos ellos poseen también los mayores valores de ubicuidad. Mientras que otros varios taxa presentan menor cantidad relativa y ubicuidad, tales como Anartrhophyllum, Senna, Lycium, Taxon A y Chusquea. Esta tendencia hacia la diversificación en el uso de leña durante el desarrollo de los conjuntos 2 y 1 (uso que está repartido por partes más o menos iguales entre varios taxa, a diferencia del conjunto 4 , donde hay una concentración marcada en las dos especies leñosas más preciadas como combustible para la zona: Adesmia y Schinus), y a la incorporación tanto de raíces como de tallos de algunas especies, considerando que la obtención de leña en general se da como una actividad a la que se responde de manera oportunista (Marston 2009) al menos en lo que al uso diario se refiere (Gélabert et al. 2011), podría deberse a una merma en la disponibilidad de Adesmia y Schinus en las cercanías del sitio por la intensificación de su uso. 
Esto parece ser consistente con el proceso de intensificación propuesto a partir del registro faunístico del sitio, que en estos niveles más modernos de la secuencia presentan un aumento en cantidad de animales pequeños, con respecto a las cantidades de guanaco recuperadas en el conjunto 4 (Neme 2007).

La presencia incluso de un taxon foráneo como Chusquea, podría estar demostrando la implementación o bien de redes de intercambio o bien de movilidad a distancia, ya que esta caña no crece naturalmente en la región sino que su distribución natural se extiende al norte de la vecina provincia de Neuquén y Chile. Junto a la Dra Llano hemos identificado este género en artefactos provenientes de otros sitios del sur mendocino como Gruta el Manzano y Alero Puesto Carrasco (próximos al río Grande, al sur de nuestra área de estudio), El Mallin (próximo al arroyo Las Aucas, tributario del Río Diamante, al norte de nuestra área de estudio), sin carbonizar y formando parte de astiles o como extremos de iniciadores de fuego (Llano 2011). También se lo ha identificado en la Gruta del Indio (en la parte baja del río Atuel) (Lagiglia 1962-1968). Estos hallazgos nos llevan a entender la presencia de esta Chusquea en el registro arqueológico de AMA-3, no como derivada de un uso combustible, sino como parte de algún tipo de instrumento (posiblemente astiles o iniciadores de fuego), en tanto que su incorporación al registro antracológico pudo haber sido producto del descarte de algún artefacto ya fuera de funcionamiento.

\section{CONCLUSIONES}

El estudio de los carbones arqueológicos procedentes de AMA-3 nos permite tener una primera aproximación para entender de qué modo las poblaciones humanas que ocuparon el sur de Mendoza, hicieron uso de los recursos maderables, específicamente de aquellos utilizados para leña. Por un lado, hemos caracterizado el registro antracológico del sitio en estudio, que es el primero de una serie de sitios arqueológicos que serán analizados en el mismo sentido en la región. La amplia secuencia temporal representada en el registro antracológico de AMA-3, y el análisis del mismo llevado a cabo en el presente trabajo permitió detectar características particulares de cada conjunto temporal e identificar hacia el Holoceno Tardío algunos rasgos que son consistentes con el proceso de intensificación regional propuesto por otros investigadores para el área a partir de otros tipos de registro arqueológico. Particularmente, el proceso de intensificación se ve reflejado en el registro antracológico de AMA-3 en primer lugar a través de la diversificación en el uso de las especies leñosas hacia el Holoceno tardío, con la consecuente incorporación de taxa no utilizados en períodos anteriores, en segundo lugar, a través de un uso repartido más o menos equitativamente entre varios géneros, en lugar de la concentración marcada en Adesmia y Schinus registrada en el Holoceno temprano; en tercer lugar, por un aprovechamiento no restringido al tallo o raíz de algunos taxa en particular sino que involucra ambos órganos de la planta hacia fines de la secuencia temporal; y por último, en la incorporación al registro de especies alóctonas hacia el Holoceno tardío.

\section{AGRADECIMIENTOS}

A la Municipalidad de Malargüe por estimular el desarrollo de esta investigación. A Adolfo Gil, Gustavo Neme y a los revisores anónimos por las sugerencias realizadas al manuscrito original, las cuales enriquecieron sustancialmente el presente trabajo. Al CONICET (PIP 0459, titular A. Capparelli) y a la Facultad de Ciencias Naturales y Museo de La Plata (Proyecto N592, titular A. Capparelli), por el soporte financiero.

\section{BIBLIOGRAFÍA}

AGÜERO BLANCH, V. 1971. La ultima ollera de Malargüe. Anales de Arqueología y Etnología. Facultad de Filosofía y Letras de UNC. Mendoza, Argentina. Tomo XXVI: 117-123

ALDENDERFER, M. 1998. Mountane Forager. Ashana and the South-Central Andean Archaic. Iowa City, University of Iowa Press

ANCIBOR, E. Y PÉREZ DE MICOU C.1995. Identification of firewood species in the archaeological record of the $\mathrm{Pa}$ tagonian Steppe. Journal of Etnobiology 15(2):189-200. 2002. Reconocimiento de especies vegetales combustibles en le registro arqueológico de la estepa patagónica. En: Pérez de Micou, C. (compiladora) Plantas y cazadores en la Patagonia. Facultad de Filosofía y Letras UBA 15-31p 
ANDREONI, D. 2010 La importancia de la colección de referencia para los análisis antracológicos, en el sur de Mendoza. Actas 5-ICES. Ed Comisión Nacional de Energía Atómica Malargüe, Argentina 30 -39p.

ANDREONI, D., Gil A., Capparelli A. 2011 Efectos de la carbonización en especies leñosas de las provincias fitogeográficas Patagónica y del Monte (Mendoza, Argentina): una perspectiva arqueológica En: Pochettino, M. y Ladio A. (ed) Traditions and transformations in Ethnobotany. Ed Cyted. San .Salvador de Jujuy. Argentina. 33-37p.

ASOUTI E. Y HATHER J., 2001. Charcoal analysis and the reconstruction of ancient woodland vegetation in the Konya Basin, south-central Anatolia, Turkey: results from the Neolithic site of Catalhöyük East. Vegetation History and Archaeobotany. 10:23-32.

BÖCHER T., HJERTING J., RAHN K., 1972. Botanical studies in the Atuel Valley area, Mendoza province, Argentina. Dansk Botanisk Arkiv 22(3) 189-358 Copenhagen. Ejnar Munksgaard

BRAADBAART F. Y POOLEL I., 2008. Morphological, chemical and physical changes during charcoalification of wood and its relevance to archaeological contexts. Journal of Archaeological Science 35(9):2434-2445

BROUGHTON M., 1994. Late Holocene resource intensification in the Sacramento Valley: the vertebrate evidence. Journal of Archaeological Science 21:501-514

CABRERA A., 1976. Regiones Fitogeográficas Argentinas. Enciclopedia Argentina de Agricultura y Jardinería, II (1):347-411 Ed Acme. Buenos Aires

CAPITANELLI R., 2005. Climatología de Mendoza. Ed Facultad de Filosofía y Letras. Universidad Nacional de Cuyo. Mendoza, Argentina

CAPPARELLI A., 2004 History of plant-people relationship and deforestation at the Hualfín Valley (Argentina), since Inca occupation (1471 AD) to the present day. Final Report to the International Foundation for Science, grand number D-3118-1, Stockholm.

2009. Intra-site comparison of the archaeoethnobotanical evidence of El Shincal: implicances to the Inka economy. En: Capparelli, A., Chevalier, A y Pique i Huerta R. (ed), La alimentación en la América precolombina y colonial: un enfoque interdisciplinario, Treballs d'Etnoarqueologia, Ed Consejo Superior de Investigación Científica, España 21-35p.

CAPPARELLI A., CASTRO A., Y M. L. CIAMPAGNA. 2009. Descripción macroscópica e identificación anatómica de un fragmento de instrumento en madera (¿arpón?) hallado en el Sitio Cueva del Negro (Costa Norte de Santa Cruz). En: Salemme M., Santiago F., Álvarez, M.,
Piana E., Vázquez M. y Mansur M. E. (ed) Arqueología de Patagonia: una mirada desde el último confín, Ed Utopías (Ushuaia) 433-444 p.

CARUSO L., MANSUR M. Y PIQUE R. 2008. Voces en el bosque el uso de los recursos vegetales entre cazadores recolectores de la zona central de Tierra del Fuego. Darwiniana 46(2):202-212

COZZO, D., 1950. Anatomía del leño secundario de las leguminosas papilionoideas Argentinas silvestres y cultivadas. Revista del Instituto Nacional de Investigación de las Ciencias Naturales Tomo 1(7):223-361

CHABAL L., 1988. Pourquoi et comment prélever les charbons de bois pour la période antique, les méthodes utilisées sur le site de Lattes (Hérault). Lattara 1:187-222.

1990. L'étude paléoécologique des sites protohistoriques à partir des charbons de bois, la question de l'unité de mesure. In: Hackens, T., Munaut, A.V., Till, C. (eds.), Wood and Archaeology, first conference. PACT, Louvain la-Neuve 189-205p.

1992. La représentativité paléoécologique des charbons de bois archéologiques issus du bois de feu. In: Vernet, J.L. (Ed.), Les charbons de bois les anciens écosystèmes et le rôle de l'Homme: Bulletin de la Société Botanique de France Actualités Botanique Tome 139:213-236.

CHRZAVZEZ J., THÉRY-PARISOT I., TERRAL J., DUCOM A. Y FIORUCCI G., 2011. Differential preservation of anthracological material and mechanical properties of wood charcoal, an experimental approach of fragmentation. En: Badal E., Carrión Y., Grau E., Macías M., y Ntinou M. 5th International Meeting of Charcoal Analysis: The charcoal as cultural and biological heritage SAGVNTVM 29-30p.

D'ANTONI H., 1983. Pollen analysis of Gruta del Indio. Quaternary of South American and Antartic Peninsula. 1:83-104

DIEGUEZ S. Y NEME G., 2003 Geochronology of the Arroyo Malo 3 site and the first human occupations in North Patagonia in the Early Holocene. En: Miotti L., Salemme M. y Flegenheimer N. (ed) Ancient Evidence for Paleo South Americans: From Where the South Winds Blows, Ed. Center for the Study of the First Americans: Texas A \& M University Press, College Station. 87-92p.

GELABERT L. P., ASOUTI E. Y ALLUÉ MARTí E., 2011. The ethnoarchaeology of firewood management in the Fang villages of Equatorial Guinea, central Africa: Implications for the interpretation of wood fuel remains from archaeological sites Journal of Anthropological Archaeology 30(3):375-384

GIL A., ZARATE M. Y NEME G., 2005. MID-HOLOCENE PALEOENVIROMENTALS AND THE ARCHAEOLO- 
GICAL RECORD OF southern Mendoza, Argentina. Quaternary International 132:81-94

GIL, A. 2006. Arqueología de La Payunia (Mendoza, Argentina): El poblamiento humano al Margen de la agricultura. BAR International Series 1477 Reino Unido

HERNÁNDEZ A., 1996. Información complementaria del departamento de Botánica para el departamento de Arqueología, referida al cathment realizado en arroyo Malo. Museo Municipal de Historia Natural de San Rafael. Mendoza, Argentina. MS

2002. Paleoetnobotánica en el Sur de Mendoza. En: Gil, A y Neme G. (ed). Entre Montañas y Desiertos Arqueología del sur de Mendoza. Ed Sociedad Argentina de Antropología. Buenos Aires. 157-180p.

IAWA 1989. List of microscopic features for hardwood identification. In E Wheeler, Baas P and Grason P (ed). IAWA Bulletin 10: 219-332

2004 List of Microscopio features for sofwood identification. In H Richter, Grosser D, Heinz I, Gasson P (ed). IAWA Journal 25(1):1-70

LAGIGLIA H., 1962/1968. Secuencia cultural del centro Oeste Argentino: Valle del Atuel y Diamante. Revista Científica de Investigaciones 1(4):131-133 Buenos Aires Argentina

LLANO C., 2005. Recursos vegetales y ocupaciones humanas: perspectivas arqueobotánicas en el Sur de Mendoza. Tesis de Licenciatura inédita. Universidad del Aconcagua, Instituto Superior San Pedro Nolasco. San Rafael Mendoza MS.

2008. El registro arqueobotánico en el sitio Arroyo Malo 3, alto valle del Atuel, Mendoza, Argentina. Intersecciones en Antropología 9:133-143.

2011. Aprovechamiento de los recursos vegetales entre las sociedades cazadoras recolectoras del sur mendocino. Tesis Doctoral Defendida en mayo del 2011. Universidad Nacional del Comaguhe. MS.

LÓPEZ M. L., CAPPARELLI A. Y NIELSEN A., 2011. Traditional post-harvest processing to make quinoa grains (Chenopodium quinoa var quinoa) apt for consumption in Northern Lipez (Potosí, Bolivia): ethnoarchaeological and archaeobotanical analices. Archaeological and Anthropological Sciences 3(1):49-70

MARCONETTO, M. B., 2002. Análisis de combustión de los sitios Alero don Santiago y de Campo Moncada. En: Pérez de Micou, C. (compiladora) Plantas y cazadores en la Patagonia. Facultad de Filosofía y Letras UBA 33-54p. 2005. Recursos forestales y el proceso de diferenciación social en tiempos prehispánicos en el valle de Ambato, Catamarca. Tesis Doctoral de la Facultad de Ciencias Naturales y Museo. Inédita
MARSTON J., 2009. Modeling wood adquisition strategies from archaeological charcoal remains. Journal of Archaeological Science. 36(10):2192-2200

MARCH R. 1992-2/3/4. Lutilisation du bois dans les foyers prehistoriques: une approche expérimentale.Les Charbons de bois les anciens Écosystèmes et le Rôle de LHomme.Colloque Monpellier Vernet J.Bulletin de la Société Botanique de France Actualités Botanique Tome 139:245-253

MILLER N., 1988. Ratios in palaeoethnobotanical analysis. En: Hastorf C. A. y Popper V. S. (ed), Current Palaeoethnobotany: analytical methods and cultural interpretations of archaeological plant remains. The University of Chicago Press, Chicago y London. 72-85 p.

MORALES M., BARBERENA R., BELARDI J.B., BORRERO L., CORTEGOSO V., DURÁN V., GUERCI A., GOÑI R., GIL A., NEME G., YACOBACCIO H. Y ZÁRATE M. 2009. Reviewing human-environment interactions in arid regions of southern South America during the past 3000 years. Palaeogeography Palaeoclimatology Palaeoecology 281(3-4): 283-295

NAVARRO, D. 2011. Ambientes Holocenos Pedemontanos y de Montaña del Sur de Mendoza (Holocene piedmont and mountain environments of southern Mendoza, Argentina). Tesis Doctoral Facultad de Ciencias Exactas y Naturales. Universidad Nacional de Mar del Plata. Inédita

NEME G., 2002. Arqueología del Alto valle del río Atuel: Modelos problemas y perspectivas en el estudio de las regiones de altura del sur de Mendoza. En: Gil, A y Neme G. (ed). Entre Montañas y Desiertos Arqueología del sur de Mendoza. Ed Sociedad Argentina de Antropología. Buenos Aires. 65-83p.

NEME G., 2007. Cazadores-recolectores de altura en los Andes Meridionales. BAR Series 1591 Reino Unido

NEME G., 2009. Un enfoque regional en cazadores-recolectores del oeste argentino: el potencial de la ecología humana". En Barberena R., Borrazzo K. y Borrero L. (ed.) Perspectivas Actuales en Arqueología Argentina. CONICET - IMHICIHU Buenos Aires, 305-326p.

NEME G. y GIL A. 2002 La explotación faunística y la frecuencia de partes esqueletarías en el registro arqueológico del sur de mendocino. En: Gil, Ay Neme G. (ed). Entre Montañas y Desiertos Arqueología del sur de Mendoza. Ed Sociedad Argentina de Antropología. Buenos Aires 141-156

NEME G. y GIL A., 2009 Human occupation and increasing midHolocene aridity. Current Anthropology 50(1):149-163.

NEME G. y A. GIL 2010 What Happened during the MidHolocene in Arid Western Argentina? A Reply to García Current Anthropology 51(3):417-419 
NEME G., MOREIRA G., ATENCIO A. y SANTIS L. 2002. El registro de microvertebrados del sitio arqueológico Arroyo Malo 3 (provincia de Mendoza, Argentina). Revista Chilena de Historia Natural 75: 409-421

NEME G., D. NAVARRO, D. ANDREONI, C. LLANO, M. GIARDINA, C. OTAOLA, F. FERNANDEZ, C. WHITLOCK, A. GIL 2011 Human subsistence changes during the late Holocene: resource over exploitation or climate change in the upper Atuel river valley, Argentina INQUA 2011 Poster

PÁEZ M., NAVARRO D., ROJO L. y GERCI A. 2010. Vegetación y paleoambiente durante el holoceno en Mendoza. En Zarate M., Gil A., Neme., (compiladores). Condiciones paleoambientales y ocupación humana durante la transición Pleistoceno- Holoceno y Holoceno en Mendoza. Ed. Sociedad Argentina de Antropología. 175-211p.

PÉREZ WINTER C., 2008. Organización Tecnológica durante el Holoceno medio en el alto valle del río Atuel, sur de Mendoza. Caso: Arroyo Malo 3. Tesis de Licenciatura, UBA. Buenos Aires. MS

PIQUE Í HUERTA, R., 1999. Producción y uso de combustible vegetal arqueológico: Una evaluación arqueológica. Treballs d Etnoarqueología № 3. Universidad Autónoma de Barcelona. Madrid.

ROIG, F., 1972. Bosquejo fisonómico de la vegetación de la provincia de Mendoza. Geología, Geomorfología, Climatología, Fitogeografía y Zoogeografía de la Provincia de Mendoza. Instituto de Investigaciones de las zonas áridas y semiáridas. Reedición especial del suplemento del Vol. XIII del Boletín de la Sociedad Argentina de Botánica de Mendoza. 49:80p.

ROIG F., MARTÍNEZ CARRETERO E. y MÉNDEZ E., 2000. Vegetación de la provincia de Mendoza. En. Abraham E. y Rodríguez F. (ed), Argentina Recursos y Problemas Ambientales de la zona Árida, Ed. Programa de Cooperación para la Investigación, Junta de Gobierno de Andalucía- Universidades y Centros de Investigación de la Región Andina, Mendoza, Argentina. 63-64p.

ROIG F. y VIDAL E., 2006/2010. Anatomía de la madera de arbustos de montaña del NW de Mendoza, Argentina. Xama 19/23:157-238.

ROSSEN J. y OLSON J., 1985. The controlled carbonization and archaeological analysis of SE U.S. wood charcoals. Journal of Field Archaeology 12(4):445-456

RUIZ LEAL A., 1972. Flora popular mendocina. Deserta III. Contribuciones del Instituto Argentino de Zonas Áridas. Mendoza, Argentina.
RUIZ LEALM A. y ROIG F., 1955. Observaciones del efecto de la nieve sobre las plantas en el arroyo Nield y valle del Atuel (Mendoza). Revista de la Facultad de Ciencias Agrarias. Universidad Nacional de Cuyo. Tomo V (1):3-55

SMART T. y HOFFMAN E., 1988. Environmental interpretation of archaeological charcoal En: Hastorf C. A. y Popper V. S. (ed), Current Palaeoethnobotany: analytical methods and cultural interpretations of archaeological plant remains. Ed The University of Chicago Press, Chicago y London, 167-205p.

SOLARI M. E., 1993. L'homme et le bois en Patagonie et Terre de Feu au cours des six deniers millénaires: recherches anthracologiques au Chili et en Argentine. Tesis Doctoral, Universidad de Montpellier, sede II, Francia

SOLARI M. E., 2000. Antracología, modo de empleo: en torno a paisajes, maderas y fogones. Revista Austral de Ciencias Sociales. 4:167-174

THIÉBAULT S., 1989 Apport de l'analyse anthracologique à la connaissance des combustibles ligneux En: Olive M., Taborin Y. (dir) Nature et fonction des foyers préhistoriques: 81-86. Colloque Internacional de Némours, 1987

THÉRY-PARISOT I., CHABAL L., y CHRZAVZEZ J., 2010. Anthracology and taphonomy, from wood gathering to charcoal analysis. A review of the taphonomic processes modifying charcoal assemblages, in archaeological contexts Palaeogeography Palaeoclimatology Palaeoecology 291:142-153

VOLKHEIMER, W. 1978. Descripción geológica de la 27 b, Cerro Sosneado. Carta geológico-económico de la República Argentina. Escala 1:200.000. Servicio Geológico Nacional, Ministerio de Economía, Buenos Aires.

WILCOX G. H., 2002. Evidence for ancient forest cover and deforestation from charcoal analysis of ten archaeological sites on the Euphrates. In: Thiébault, S., (Ed.), Charcoal Analysis: Methodological Approaches, Palaeoecological Results and Wood Uses: Proceedings of the Second International Meeting of Anthracology, Paris, September 2000. Archaeopress, Oxford, pp. 141-145

ZÁRATE M., 2002. Los ambientes del Tardiglacial y Holoceno en Mendoza. En: Gil, A y Neme G. (ed). Entre Montañas y Desiertos Arqueología del sur de Mendoza. Ed Sociedad Argentina de Antropología. Buenos Aires. Argentina 9-42 p.

ZÁRATE M., GIL A. y NEME G. 2010. Integrando los registros: generalizaciones, alcances y limitaciones. En Zarate M., Gil A., Neme., (compiladores). Condiciones paleoambientales y ocupación humana durante la transición Pleistoceno- Holoceno y Holoceno en Mendoza. Ed. Sociedad Argentina de Antropología 309-333p 